Yuxi Meng, Xinrui Zhang, and Xiaoming $\mathrm{He}^{\star}$

\title{
Ground state solutions for a class of fractional Schrodinger-Poisson system with critical growth and vanishing potentials
}

https://doi.org/10.1515/anona-2020-0179

Received November 24, 2020; accepted March 9, 2021.

Abstract: In this paper, we study the fractional Schrödinger-Poisson system

$$
\begin{cases}(-\Delta)^{s} u+V(x) u+K(x) \phi|u|^{q-2} u=h(x) f(u)+|u|^{2_{s}^{*}-2} u, & \text { in } \mathbb{R}^{3}, \\ (-\Delta)^{t} \phi=K(x)|u|^{q}, & \text { in } \mathbb{R}^{3},\end{cases}
$$

where $s, t \in(0,1), 3<4 s<3+2 t, q \in\left(1,2_{s}^{\star} / 2\right)$ are real numbers, $(-\Delta)^{s}$ stands for the fractional Laplacian operator, $2_{s}^{\star}:=\frac{6}{3-2 s}$ is the fractional critical Sobolev exponent, $K, V$ and $h$ are non-negative potentials and $V, h$ may be vanish at infinity. $f$ is a $C^{1}$-function satisfying suitable growth assumptions. We show that the above fractional Schrödinger-Poisson system has a positive and a sign-changing least energy solution via variational methods.

Keywords: Fractional Schrödinger-Poisson system; Potential vanishing at infinity; Variational methods.

MSC: 35J20, 35A15, 35B33

\section{Introduction}

In this paper, we study the existence of positive and sign-changing least energy solutions for the following fractional Schrödinger-Poisson system

$$
\begin{cases}(-\Delta)^{s} u+V(x) u+K(x) \phi|u|^{q-2} u=h(x) f(u)+|u|^{2_{s}^{*}-2} u, & \text { in } \mathbb{R}^{3}, \\ (-\Delta)^{t} \phi=K(x)|u|^{q}, & \text { in } \mathbb{R}^{3},\end{cases}
$$

where $s, t \in(0,1), 3<4 s<3+2 t, q \in\left(1,2_{s}^{*} / 2\right)$ are real numbers, $2_{s}^{\star}:=\frac{6}{3-2 s}$ is the fractional critical Sobolev exponent. $(-\Delta)^{s}$ is the fractional Laplacian operator defined as

$$
(-\Delta)^{s} u(x)=C_{s} \text { P.V. } \int_{\mathbb{R}^{3}} \frac{u(x)-u(y)}{|x-y|^{3+2 s}} d y, \quad x \in \mathbb{R}^{3},
$$

where P.V. is a commonly used abbreviation for in the principal value sense, and $C_{s}$ is a suitable normalization constant.

We notice that, when $s=t=1$ and $V(x) \equiv 1$, Ghergu and Singh [10] considered problem (1.1) with Choquard nonlinearity,

$$
\begin{cases}-\Delta u+u+K(x) \phi|u|^{q-2} u=\left(I_{\alpha} \star|u|^{p}\right)|u|^{p-2} u, & \text { in } \mathbb{R}^{3} \\ -\Delta \phi=K(x)|u|^{q}, & \text { in } \mathbb{R}^{3},\end{cases}
$$

Yuxi Meng, College of Science, Minzu University of China, Beijing 100081, China, E-mail: 1161881981@qq.com Xinrui Zhang, College of Science, Minzu University of China, Beijing 100081, China, E-mail: 1343157482@qq.com *Corresponding Author: Xiaoming He, College of Science, Minzu University of China, Beijing 100081, China, E-mail: xmhe923@muc.edu.cn 
where $I_{\alpha}(x)=|x|^{-(3-\alpha)}, \alpha \in(0,3)$, is the Ruiz potential. The authors discussed the existence of a ground state solution by using a variational approach. They also used a Pohozaev type identity to derive conditions in terms of $p, q, N, \alpha$ and $K$ for which no solutions exist.

Formally, the system

$$
\begin{cases}-\Delta u+V(x) u+K(x) \phi|u|^{q-2} u=|u|^{2 p-2} u, & \text { in } \mathbb{R}^{3} \\ -\Delta \phi=K(x)|u|^{q}, & \text { in } \mathbb{R}^{3},\end{cases}
$$

can be seen as a formal limit of (1.2) as $\alpha \rightarrow 0$. The classical nonlinear Schrödinger equation

$$
i \psi_{t}+\Delta \psi+V_{\text {ext }}(x) \psi+\left(I_{2}{ }^{\star}|\psi|^{2}\right) \psi-|\psi|^{p-2} \psi=0, \quad(t, x) \in \mathbb{R}^{+} \times \mathbb{R}^{3} .
$$

is used as an approximation to the Hartree-Fock model of a quantum many body-system of electrons under the presence of an external potential $V_{\text {ext }}$. In such a setting, (1.4) and its stationary counterpart bear the name of Schrödinger-Poisson-Slater, Schrödinger-Poisson- $X_{\alpha}$, or Maxwell-Schrödinger-Poisson equation. The convolution term in (1.4) represents the Coulombic repulsion between the electrons. The local term $|u|^{2 p-2} u$ was introduced by Slater as a local approximation of the exchange potential in the Hartree-Fock model. We refer to $[6,19,28]$ for more applied background for $(1.4)$.

When $s=t=1$ and $q=2$, system (1.1) reduces to the following Schrödinger-Poisson system

$$
\begin{cases}-\Delta u+V(x) u+K(x) \phi(x) u=f(x, u), & \text { in } \mathbb{R}^{3} \\ -\Delta \phi=K(x) u^{2}, & \text { in } \mathbb{R}^{3},\end{cases}
$$

which plays a great role in looking for solitary waves of the nonlinear stationary Schrödinger equations interacting the electrostatic field. It also appears in physics such as quantum mechanics models[22], semiconductor theory[5] and so on. The nonlinearity $f$ represents the particles interacting with each other. The term $K \phi u$ represents the interaction with the electric field. We refer the interesting readers to[2] and the references therein for more details about the mathematical and physical backgrounds. Recently, there are many studies on the existence of solutions to (1.5) by using the methods of nonlinear analysis. In [47], the authors established the existence of a positive solution of (1.5) with a critical nonlinearity $K(x)|u|^{2^{*}-2} u+\mu Q(x)|u|^{q-2} u$, by using the concentration-compactness principle of P. L. Lions and methods of Brezis and Nirenberg. Later, He and Zou [12] studied the existence and concentration behavior of ground state solutions for (1.5) with a critical nonlinearity and double parameters perturbation, namely

$$
\begin{cases}-\varepsilon^{2} \Delta u+V(x) u+\phi(x) u=f(u)+u^{2^{*}-1}, & \text { in } \mathbb{R}^{3} \\ -\varepsilon^{2} \Delta \phi=u^{2}, u(x)>0, & \text { in } \mathbb{R}^{3} .\end{cases}
$$

Under some suitable conditions on the nonlinearity $f$ and the potential $V$, they proved that for $\varepsilon$ small, (1.6) has a ground state solution concentrating around global minimum of the potential $V$ in the semi-classical limit. For more results on the multiple nontrivial solutions, infinitely many nontrivial solutions, ground state solutions, positive solutions, semiclassical state solutions and sign-changing solutions for (1.5) under various conditions on $V, K$ and $f$, we refer to $[4,8,14,15,23,25,29-31,38,39,41,46]$ and references therein.

In recent years, there is an increasing interest in the studying for the fractional Schrödinger-Poisson system (1.1) with $q=2$, i.e.,

$$
\begin{cases}(-\Delta)^{s} u+V(x) u+K(x) \phi(x) u=f(x, u), & \text { in } \mathbb{R}^{3} \\ (-\Delta)^{t} \phi=K(x) u^{2}, & \text { in } \mathbb{R}^{3}\end{cases}
$$

where $s, t \in(0,1)$. For example, in [34], Teng considered (1.7) with $f(x, u)=\mu|u|^{p-1} u+|u|^{2_{s}^{*}-2} u, 1<p<$ $2_{s}^{\star}-1$. The author used the method of Pohozaev-Nehari manifold and global compactness Lemma to obtain a ground state solutions. In [33], Teng studied the existence of ground states to (1.7) with subcritical growth, i.e., $f(x, u)=|u|^{p-1} u$, by using a monotonicity trick and global compactness principle.

In [21], Murcia and Siciliano considered the following semiclassical problem

$$
\begin{cases}\varepsilon^{2 s}(-\Delta)^{s} u+V(x) u+\phi u=g(u) & \text { in } \mathbb{R}^{3}, \\ \varepsilon^{2 \theta}(-\Delta)^{\alpha / 2} \phi=\gamma^{\alpha} u^{2}, & \text { in } \mathbb{R}^{3},\end{cases}
$$


and established the multiplicity of solutions for small $\varepsilon$ via Ljusternik-Schnirelmann category theory, where $g$ is subcritical at infinity. In [43], the authors obtained the multiplicity of solution for (1.8) when $g$ is critical growth. For more results on the concentration behavior of semiclassical solutions for (1.8), we refer to [18, 42, 44]. For the existence results on ground state solutions, sign-changing solutions for (1.7), we refer to [17, 21, $37,45]$ and the references therein.

When $q \neq 2$, we only note that in [36], Teng and Agarwal studied the existence and non-existence of ground state states via variational method, for (1.1) with the subcritical Choquard nonlinearity $\left(I_{\alpha}\right.$ * $\left.|u|^{p}\right)|u|^{p-2} u$, that is,

$$
\begin{cases}(-\Delta)^{s} u+V(x) u+K(x) \phi|u|^{q-2} u=\left(I_{\alpha} \star|u|^{p}\right)|u|^{p-2} u, & \text { in } \mathbb{R}^{3}, \\ (-\Delta)^{t} \phi=K(x)|u|^{q}, & \text { in } \mathbb{R}^{3},\end{cases}
$$

where $\frac{3+\alpha}{3}<p<\frac{3+\alpha}{3-2 s}, 1<q<p$. The assumption on $V$ in [32] was formulated as follows:

$\left(V_{0}\right) \quad V(x) \in C\left(\mathbb{R}^{3}, \mathbb{R}\right), \lim _{|x| \rightarrow \infty} V(x)=V_{\infty}>0 ;$

$\left(V_{1}\right) \quad 0<V_{0}=\inf _{x \in \mathbb{R}^{3}} V(x) \leq V_{\infty}, \forall x \in \mathbb{R}^{3}$;

$\left(V_{2}\right) \quad V(x) \geq V_{\infty}, \forall x \in \mathbb{R}^{3}, W(x):=V(x)-V_{\infty} \in L^{\frac{3}{2 s}}\left(\mathbb{R}^{3}\right)$ and $V(x)>V_{\infty}$ on a positive measure set.

Under some suitable conditions on $p, q$ and $K$, the authors proved the existence of a nonnegative ground states to (1.9), by using the hypotheses $\left(V_{0}\right),\left(V_{1}\right)$ and a minimization argument; and the existence of bound states by employing the hypotheses $\left(V_{0}\right),\left(V_{2}\right)$ and a linking theorem.

In light of the above cited works, the main purpose of this paper is to study the existence of (signchanging) solutions of (1.1) under the simultaneous presence of nonlinearities having critical growth and potentials $V, h$ are permitted to vanishing asymptotically as $|x| \rightarrow \infty$, without any symmetry assumptions made on $V, h$. To the best of our knowledge, there is not any result for the existence of positive and signchanging least energy solutions for the fractional Schrödinger-Poisson system (1.1) in the existing literature.

In order to state the main results, we introduce the assumptions on the potentials $K, V$ and $h$. The nonnegative function $K$ satisfies following condition:

(K) $K \in L^{r}\left(\mathbb{R}^{3}\right) \cap L^{\infty}\left(\mathbb{R}^{3}\right) \backslash\{0\}$ for some $r \in[6 /(3+2 t-q(3-2 s)), \infty)$.

The functions $V, h: \mathbb{R}^{3} \rightarrow \mathbb{R}$, are continuous and we say that $(V, h) \in \mathcal{H}$ if the following conditions hold true:

$\left(V h_{0}\right) V(x), h(x)>0$ for all $x \in \mathbb{R}^{3}$ and $h(x) \in L^{\infty}\left(\mathbb{R}^{3}\right)$;

$\left(V h_{1}\right)$ If $\left\{A_{n}\right\}_{n \in \mathbb{N}} \subset \mathbb{R}^{3}$ is a sequence of Borel sets such that the Lebesgue measure $m\left(A_{n}\right)$ is less than or equal to $R$, for all $n \in \mathbb{N}$ and some $R>0$, we have

$$
\lim _{r \rightarrow+\infty} \int_{A_{n} \cap B_{r}^{c}(0)} h(x) d x=0,
$$

uniformly in $n \in \mathbb{N}$, where $B_{r}^{c}(0):=\mathbb{R}^{3} \backslash B_{r}(0)$.

$\left(V h_{2}\right)$ Either $\frac{h(x)}{V(x)} \in L^{\infty}\left(\mathbb{R}^{3}\right)$; or

$\left(V h_{3}\right)$ there exists $p \in\left(2 q, 2_{s}^{\star}\right)$, such that

$$
\frac{h(x)}{[V(x)]^{(2)-p) /\left(2_{s}^{*}-2\right)}} \rightarrow 0 \text { as }|x| \rightarrow+\infty,
$$

For what concerns the nonlinearity $f$, we assume that $f$ fulfills the following conditions:

$\left(f_{1}\right) \lim _{|t| \rightarrow 0} \frac{f(t)}{|t|^{2 q-1}}=0$ if $\left(V h_{2}\right)$ holds; or

$\left(\widetilde{f}_{1}\right) \lim _{|t| \rightarrow 0} \frac{f(t)}{|t|^{p-1}}<+\infty$ if $\left(V h_{3}\right)$ holds, for some $p \in\left(2 q, 2_{s}^{\star}\right)$;

$\left(f_{2}\right) \lim _{|t| \rightarrow+\infty} \frac{F(t)}{t^{2 q}}=+\infty$, where $F(t):=\int_{0}^{t} f(\tau) d \tau$;

$\left(f_{3}\right) \frac{f(t)}{|t|^{2 q-1}}$ is strictly increasing function of $t \in \mathbb{R} \backslash\{0\}$; 
$\left(f_{4}\right)$ there exist $\mu, v \in\left(2 q, 2_{s}^{*}\right)$ and $\lambda>0$, such that $F(t) \geq \lambda t^{\mu}, \forall t \geq 0$, and

$$
\lim _{|t| \rightarrow+\infty} \frac{f(t)}{|t|^{v-1}}=0 .
$$

Remark 1.1. From the assumption about function $h$, there exists $x_{0} \in \mathbb{R}^{3}$ such that $h\left(x_{0}\right)>0$, without loss of generality, we may assume that $x_{0}=0$. So, by the continuous of $h$, there exists small $r_{0}>0$ such that $\min _{|x| \leq r_{0}} h(x):=h_{0}>0$.

The main results of this paper can be stated as follows.

Theorem 1.1. Let $\frac{3+2 t}{2(3-2 s)}<q<\frac{3}{3-2 s}$ and assume that $(K)$ holds, $(V, h) \in \mathcal{H}$, and $f$ satisfies either $\left(f_{1}\right)$ or $\left(\widetilde{f}_{1}\right)$ and $\left(f_{2}\right)-\left(f_{4}\right)$, then problem (1.1) possesses a positive ground state solution.

Theorem 1.2. Let $\max \left\{2, \frac{3+2 t}{2(3-2 s)}\right\}<q<\frac{3}{3-2 s}$, and assume that $(K)$ holds, $(V, h) \in \mathcal{H}$, and $f$ satisfies either $\left(f_{1}\right)$ or $\left(\widetilde{f}_{1}\right)$ and $\left(f_{2}\right)-\left(f_{4}\right)$. Moreover,

$$
\max \left\{\frac{9 q-3-2 s q-2 t}{q(3-2 s)}, \frac{3+2 s}{3-2 s}, p, \mu\right\}<v<2_{s}^{\star} .
$$

Then problem (1.1) has a sign-changing least energy solution.

Remark 1.2. We note that, in the case $K(x) \equiv 0$, problem (1.1) reduces to the simple equation $(-\Delta)^{s} u+V(x) u=$ $h(x) f(u)+|u|^{2_{s}^{*}-2} u$, studied by do Ó, Miyagaki and Squassina in [9] where they obtained the existence of nontrivial solutions for this single fractional Schrödinger equation using the hypotheses $\left(V h_{0}\right)-\left(V h_{3}\right)$. When $K(x) \neq \equiv$, owing to the nonlocal property of $(-\Delta)^{s}$ and the nonlocal term $K(x) \phi_{u}^{t}$ (see Sect. 2) the study of (1.1) becomes more involved and harder to handle, the usual method for finding sign-changing solutions is not applicable directly. In addition, in the critical case the invariance by dilations of $\mathbb{R}^{3}$ has to be considered. While dealing with problem (1.1), alike the local case: $s=t=1$, we have to overcome the well known double lack of compactness caused by the critical nonlinearity and the unbounded domain $\mathbb{R}^{3}$, which prevents us from using the variational methods in a standard way. To recover the compactness of Palais-Smale sequences, we need to exploit hypotheses $\left(V h_{0}\right)-\left(V h_{3}\right)$ to obtain the Sobolev compactness embedding for the weighted space, see Proposition 2.3 below, and some new delicate estimates concerning the nonlocal term and the critical nonlinearity. We note that in [47], the authors avoided the lack of compactness of the Sobolev embedding by looking for solutions of (1.5) in the subspace of radial functions of $H^{1}\left(\mathbb{R}^{3}\right)$, which is usually denoted by $H_{r}^{1}\left(\mathbb{R}^{3}\right)$, since in this case the embedding $H_{r}^{1}\left(\mathbb{R}^{3}\right) \rightarrow L^{s}\left(\mathbb{R}^{3}\right)(2<s<6)$ is compact. For the results on the classical Schrödinger equations with vanishing potentials, we refer to $[1,3]$ and the references therein.

This paper is organized as follows. In Section 2 we present some facts about the involved fractional Sobolev spaces and technical results, which are useful to prove the main results. In Section 3, we show the existence of positive solution for problem (1.1) and complete the proof of Theorem 1.1. In Section 4, we show the existence of sign-changing solutions of problem (1.1), and prove Theorem 1.2.

\section{Notations:}

- $B_{R}(y)$ denotes the ball centered at $y$ with radius $R ; B_{R}:=B_{R}(0)$.

- $L^{p}(\Omega), 1 \leq p \leq \infty, \Omega \subseteq \mathbb{R}^{3}$, are the usual spaces with norm denoted by $\|\cdot\|_{L^{p}(\Omega)}$; if $\Omega=\mathbb{R}^{3}$, we simply write $\|\cdot\|_{p}$.

- Positive constants whose exact values are not important in the relevant arguments, and that may vary from line to line, are generally denoted by $C$ or $C_{i}$, where $i \in \mathbb{N}$. 


\section{Variational setting and preliminary lemmas}

In this section we outline the variational framework for studying problem (1.1) and present some preliminary results, which are useful for the proof of the main results in Sections 3, 4.

We first fix some notations as follows. The homogeneous fractional Sobolev space $\mathcal{D}^{s, 2}\left(\mathbb{R}^{3}\right)(s \in(0,1))$ is defined by

$$
\mathcal{D}^{s, 2}\left(\mathbb{R}^{3}\right)=\left\{u \in L^{2_{s}^{*}}\left(\mathbb{R}^{3}\right): \frac{|u(x)-u(y)|}{|x-y|^{\frac{3}{2}+s}} \in L^{2}\left(\mathbb{R}^{3} \times \mathbb{R}^{3}\right)\right\},
$$

which is the completion of $\mathcal{C}_{0}^{\infty}\left(\mathbb{R}^{3}\right)$ under the norm

$$
\|u\|_{\mathcal{D}^{s, 2\left(\mathbb{R}^{3}\right)}}=\left\|(-\Delta)^{\frac{s}{2}} u\right\|_{L^{2}\left(\mathbb{R}^{3}\right)}=\left(\iint_{\mathbb{R}^{6}} \frac{|u(x)-u(y)|^{2}}{|x-y|^{3+2 s}} d x d y\right)^{\frac{1}{2}} .
$$

The embedding $\mathcal{D}^{s, 2}\left(\mathbb{R}^{3}\right) \rightarrow L^{2_{s}^{*}}\left(\mathbb{R}^{3}\right)$ is continuous and the best constant $\mathcal{S}_{s}$ can be defined as

$$
\mathcal{S}_{s}=\inf _{u \in \mathcal{D}^{s, 2\left(\mathbb{R}^{3}\right) \backslash\{0\}}}=\frac{\int_{\mathbb{R}^{3}}\left|(-\Delta)^{\frac{s}{2}} u\right|^{2} d x}{\left(\int_{\mathbb{R}^{3}}|u(x)|^{2_{s}^{\star}} d x\right)^{\frac{2}{2_{s}^{*}}}} .
$$

The fractional Sobolev space $H^{s}\left(\mathbb{R}^{3}\right)$ is defined by

$$
H^{s}\left(\mathbb{R}^{3}\right)=\left\{u \in L^{2}\left(\mathbb{R}^{3}\right): \frac{|u(x)-u(y)|}{|x-y|^{\frac{3}{2}+s}} \in L^{2}\left(\mathbb{R}^{3} \times \mathbb{R}^{3}\right)\right\}
$$

endowed with the norm

$$
\|u\|_{H^{s}\left(\mathbb{R}^{3}\right)}^{2}=[u]^{2}+\|u\|_{2}^{2}=\iint_{\mathbb{R}^{6}} \frac{|u(x)-u(y)|^{2}}{|x-y|^{3+2 s}} d x d y+\int_{\mathbb{R}^{3}}|u|^{2} d x,
$$

where

$$
[u]^{2}=\iint_{\mathbb{R}^{6}} \frac{|u(x)-u(y)|^{2}}{|x-y|^{3+2 s}} d x d y .
$$

We define the work space for (1.1) by

$$
H=\left\{u \in H^{s}\left(\mathbb{R}^{3}\right): \int_{\mathbb{R}^{3}} V(x)|u|^{2} d x<+\infty\right\}
$$

with the norm

$$
\|u\|^{2}=[u]^{2}+\int_{\mathbb{R}^{3}} V(x)|u|^{2} d x=\iint_{\mathbb{R}^{6}} \frac{|u(x)-u(y)|^{2}}{|x-y|^{3+2 s}} d x d y+\int_{\mathbb{R}^{3}} V(x)|u|^{2} d x .
$$

We recall the following well-known Hardy-Littlewood-Sobolev inequality as follows:

Proposition 2.1. ([16]) Let $t, r>1$, and $0<\mu<3$ with $\frac{1}{t}+\frac{\mu}{3}+\frac{1}{r}=2, f \in L^{t}\left(\mathbb{R}^{3}\right)$ and $h \in L^{r}\left(\mathbb{R}^{3}\right)$. There exists a sharp constant $C(t, \mu, r)$, independent of $f$ and $h$, such that

$$
\iint_{\mathbb{R}^{6}} \frac{f(x) h(y)}{|x-y|^{\mu}} d x d y \leq C(t, \mu, r)|f|_{t}|h|_{r}
$$

Now, we are going to reduce system (1.1) to a single equation. To this aim, for any $u \in H^{s}\left(\mathbb{R}^{3}\right)$, we define the $\operatorname{map} \mathcal{L}_{u}: D^{t, 2}\left(\mathbb{R}^{3}\right) \rightarrow \mathbb{R}$ as

$$
\mathcal{L}_{u}(v)=\int_{\mathbb{R}^{3}} K(x)|u|^{q} v d x .
$$

Using the Hölder's inequality, $(K)$ and the Sobolev embedding inequality, we get

$$
\left|\mathcal{L}_{u}(v)\right| \leq\|K\|_{r}\|u\|_{6 q r /(r(3+2 t)-6)}^{q}\|v\|_{2_{t}^{*}} \leq S_{S}^{-\frac{1}{2}}\|K\|_{r}\|u\|_{6 q r /(r(3+2 t)-6)}^{q}\|v\|_{D^{t, 2}} .
$$


Here we have used the fact $2<6 q /(3+2 t)<6 q r /[r(3+2 t)-6] \leq 2_{s}^{\star}$, and $r \in[6 /(3+2 t-q(3-2 s)), \infty)$. Hence, $\mathcal{L}_{u}$ is a linear and continuous map on $D^{t, 2}\left(\mathbb{R}^{3}\right)$. Therefore, by the Lax-Milgram theorem, there exists a unique $\phi_{u}^{t} \in \mathcal{D}^{t, 2}\left(\mathbb{R}^{3}\right)$ such that $\mathcal{L}_{u}(v)=\left\langle\phi_{u}^{t}, v\right\rangle$ for all $v \in \mathcal{D}^{t, 2}\left(\mathbb{R}^{3}\right)$. In other words, $\phi_{u}^{t}$ is the weak solution of

$$
(-\Delta)^{t} \phi_{u}^{t}=K(x)|u|^{q}, \quad x \in \mathbb{R}^{3}
$$

and the representation formula holds

$$
\phi_{u}^{t}(x)=C_{t} \int_{\mathbb{R}^{3}} \frac{K(y)|u(y)|^{q}}{|x-y|^{3-2 t}} d y, \quad x \in \mathbb{R}^{3},
$$

which is called $t$-Riesz potential, which can be rewritten as

$$
\phi_{u}^{t}(x)=I_{2 t} \star\left(K|u|^{q}\right), \quad C_{t}=\pi^{-\frac{3}{2}} 2^{-2 t} \frac{\Gamma\left(\frac{3-2 t}{2}\right)}{\Gamma(t)} .
$$

In the sequel, we often omit the constant $C_{t}$ for convenience. Substituting $\phi_{u}^{t}$ in (1.1), we have the following single fractional Schrödinger equation

$$
(-\Delta)^{s} u+V(x) u+K(x) \phi_{u}^{t} u=h(x) f(u)+|u|^{2_{s}^{*}-2} u, \text { in } \mathbb{R}^{3}
$$

So, the energy functional $I: H \rightarrow \mathbb{R}$ associated with (2.4),

$$
\begin{aligned}
I(u)= & \frac{1}{2} \int_{\mathbb{R}^{3}}\left|(-\Delta)^{\frac{s}{2}} u\right|^{2} d x+\frac{1}{2} \int_{\mathbb{R}^{3}} V(x) u^{2} d x+\frac{1}{2 q} \int_{\mathbb{R}^{3}} K(x) \phi_{u}^{t}|u|^{q} d x \\
& -\int_{\mathbb{R}^{3}} h(x) F(u) d x-\frac{1}{2_{s}^{\star}} \int_{\mathbb{R}^{3}}|u|^{2_{s}^{\star}} d x
\end{aligned}
$$

is well defined on $H$. It is easy to see that $I$ is of class $C^{1}$ and for any critical point of $I$ is a weak solution of (2.4).

We shall seek the ground state solution of problem (2.4) on the Nehari manifold

$$
\mathcal{N}:=\left\{u \in H \backslash\{0\}:\left\langle I^{\prime}(u), u\right\rangle=0\right\},
$$

and define the following minimization problem:

$$
c_{0}:=\inf _{u \in \mathcal{N}} I(u)
$$

We call $u$ is a least energy sign-changing solution to problem (2.4) if $u$ is a solution of problem (2.5) with $u^{ \pm} \neq 0$ and

$$
I(u)=\inf \left\{I(v): v^{ \pm} \neq 0, I^{\prime}(v)=0\right\}
$$

where $u^{+}(x):=\max \{u(x), 0\}, u^{-}(x):=\min \{u(x), 0\}$. Since there exist nonlocal terms $(-\Delta)^{s} u$ and $\phi_{u}^{t}$ in (2.4), we have

$$
\int_{\mathbb{R}^{3}}(-\Delta)^{\frac{s}{2}} u^{+}(-\Delta)^{\frac{s}{2}} u^{-} d x>0 \text { and } \int_{\mathbb{R}^{3}} K(x) \phi_{u^{+}}^{t}\left|u^{-}\right|^{2} d x>0
$$

for $u^{ \pm} \neq 0$, a direct computation yields that

$$
\begin{aligned}
& I(u)>I\left(u^{+}\right)+I\left(u^{-}\right), \\
& \left\langle I^{\prime}(u), u^{+}\right\rangle>\left\langle I^{\prime}\left(u^{+}\right), u^{+}\right\rangle, \text {and }\left\langle I^{\prime}(u), u^{-}\right\rangle>\left\langle I^{\prime}\left(u^{-}\right), u^{-}\right\rangle .
\end{aligned}
$$

So the methods to obtain sign-changing solutions of the local problems and to estimate the energy of the sign-changing solutions seem not suitable for our nonlocal one (2.4). In order to get a sign-changing solution for problem (2.4), we will consider the following minimization problem:

$$
\begin{gathered}
m:=\inf \{I(u): u \in \mathcal{M}\}, \\
\mathcal{M}:=\left\{u \in H: u^{ \pm} \neq 0,\left\langle I^{\prime}(u), u^{+}\right\rangle=\left\langle I^{\prime}(u), u^{-}\right\rangle=0\right\} .
\end{gathered}
$$


Finally, we introduce the weighted Banach space:

$$
L_{h}^{p}\left(\mathbb{R}^{3}\right)=\left\{u: \mathbb{R}^{3} \rightarrow \mathbb{R} \text { measurable and } \int_{\mathbb{R}^{3}} h(x)|u|^{p} d x<\infty\right\},
$$

equipped with the norm:

$$
\|u\|_{L_{h}^{p}}=\left(\int_{\mathbb{R}^{3}} h(x)|u|^{p} d x\right)^{\frac{1}{p}}
$$

Proposition 2.2. ([9]) Assume that $(V, h) \in \mathcal{H}$ and $f$ satisfies either $\left(f_{1}\right)$ or $\left(\widetilde{f}_{1}\right)$ and $\left(f_{2}\right)-\left(f_{4}\right)$. Let $\left\{u_{n}\right\}_{n \in \mathbb{N}}$ be a sequence such that $u_{n} \rightarrow u$ in $H$, then

$$
\lim _{n \rightarrow+\infty} \int_{\mathbb{R}^{3}} h(x) F\left(u_{n}\right) d x=\int_{\mathbb{R}^{3}} h(x) F(u) d x
$$

and

$$
\lim _{n \rightarrow+\infty} \int_{\mathbb{R}^{3}} h(x) f\left(u_{n}\right) u_{n} d x=\int_{\mathbb{R}^{3}} h(x) f(u) u d x
$$

Proposition 2.3. ([9]) Assume that $(V, h) \in \mathcal{H}$. Then, $H$ is compactly embedded in $L_{h}^{r}\left(\mathbb{R}^{3}\right)$ for all $r \in\left(2,2_{s}^{\star}\right)$ if $\left(V h_{2}\right)$ holds. If $\left(V h_{3}\right)$ holds, $H$ is compactly embedded in $L_{h}^{p}\left(\mathbb{R}^{3}\right)$.

Proposition 2.4. Assume that $(K)$ holds, then for any $u \in H^{s}\left(\mathbb{R}^{3}\right)$, there exists a unique $\phi_{u}^{t} \in D^{t, 2}\left(\mathbb{R}^{3}\right)$ solution of the equation $(-\Delta)^{t} \phi=K(x)|u|^{q}$. Such a solution is nonnegative and has the following representation:

$$
\phi_{u}^{t}=I_{2 t}{ }^{\star}\left(K|u|^{q}\right) .
$$

Moreover, (i) there exist two constants $C, C_{1}>0$ such that

$$
\left\|\phi_{u}^{t}\right\|_{D^{t, 2\left(\mathbb{R}^{3}\right)}} \leq C\|K\|_{r}\|u\|_{6 q r /(r(3+2 t)-6)}^{q}, \quad \int_{\mathbb{R}^{3}} K(x) \phi_{u}^{t}|u|^{q} d x \leq C_{1}\|K\|_{r}^{2}\|u\|_{6 q r /(r(3+2 t)-6)}^{2 q} .
$$

(ii) If $u_{n} \rightarrow u$ in $H^{s}\left(\mathbb{R}^{3}\right)$, then

(a) $\lim _{n \rightarrow \infty} \int_{\mathbb{R}^{3}} K(x) \phi_{u_{n}}^{t}|u|_{n}^{q} d x=\int_{\mathbb{R}^{3}} K(x) \phi_{u}^{t}|u|^{q} d x$;

(b) $\lim _{n \rightarrow \infty} \int_{\mathbb{R}^{3}} K(x) \phi_{u_{n}}^{t}\left|u_{n}\right|^{q-2} u_{n} v d x=\int_{\mathbb{R}^{3}} K(x) \phi_{u}^{t}|u|^{q-2} u v d x$ for any $v \in H^{s}\left(\mathbb{R}^{3}\right)$;

Proof. (i) follows from the definition of $\phi_{u}^{t}$ and (H).

(ii) First of all, we claim that

$$
\lim _{n \rightarrow \infty} \int_{\mathbb{R}^{3}} K(x) \phi_{u_{n}}^{t}\left(\left|u_{n}\right|^{q}-|u|^{q}\right) d x=0 .
$$

In fact, using Proposition 2.1, we have

$$
\begin{aligned}
\left|\int_{\mathbb{R}^{3}} K(x) \phi_{u_{n}}^{t}\left(\left|u_{n}\right|^{q}-|u|^{q}\right) d x\right| & =\left|\int_{\mathbb{R}^{3}} \int_{\mathbb{R}^{3}} \frac{K(x) K(y)\left|u_{n}(y)\right|^{q}\left(\left|u_{n}\right|^{q}(x)-|u|^{q}(x)\right)}{|x-y|^{3-2 t}} d x d y\right| \\
& \leq C\left\|u_{n}\right\|_{6 q /(3+2 t)}^{q}|| K\left(\left|u_{n}\right|^{q}-|u|^{q}\right) \|_{6 /(3+2 t)} \\
& \leq C\left(\left.\int_{\mathbb{R}^{3}}|K|^{6 /(3+2 t)}|| u_{n}\right|^{q}-\left.|u|^{q}\right|^{6 /(3+2 t)} d x\right)^{\frac{3+2 t}{6}} .
\end{aligned}
$$

For any $\delta>0$, using Young inequality, one has

$$
\begin{aligned}
\left.|K|^{6 /(3+2 t)}|| u_{n}\right|^{q}-\left.|u|^{q}\right|^{6 /(3+2 t)} & \leq C|K|^{6 /(3+2 t)}\left|u_{n}\right|^{6 q /(3+2 t)}+C|K|^{6 /(3+2 t)}|u|^{6 q /(3+2 t)} \\
& \leq \delta\left|u_{n}\right|^{\frac{6 q r}{r(3+2 t)-6}}+C_{\delta}|K|^{r}+\delta|u|^{\frac{6 q r}{r(3+2 t)-6}} \\
& =\delta\left(\left|u_{n}\right|^{\frac{6 q r}{(3+2 t)-6}}-|u|^{\frac{6 q r}{r(3+2 t)-6}}\right)+C_{\delta}|K|^{r}+2 \delta|u|^{\frac{6 q r}{r(3+2 t)-6}} .
\end{aligned}
$$


Set

$$
G_{\delta, n}(x):=\max \left\{|K|^{6 /(3+2 t)}\left(\left|u_{n}\right|^{q}-|u|^{q}\right)^{6 /(3+2 t)}-\delta\left(\left|u_{n}\right|^{\frac{6 q r}{r(3+2 t)-6}}-|u|^{\frac{6 q r}{r(3+2 t)-6}}\right), 0\right\}
$$

Then

$$
G_{\delta, n}(x) \rightarrow 0 \text { a.e. } x \in \mathbb{R}^{3}
$$

and

$$
0 \leq G_{\delta, n}(x) \leq C_{\delta}|K|^{r}+2 \delta|u|^{\frac{6 q r}{r(3+2 t)-6}} \in L^{1}\left(\mathbb{R}^{3}\right) .
$$

Here we have used the fact $2<6 q /(3+2 t)<6 q r /[r(3+2 t)-6] \leq 2_{s}^{\star}$, and $r \in[6 /(3+2 t-q(3-2 s))$, $\infty)$. Hence, by the Lebesgue dominated convergence theorem, one has

$$
\lim _{n \rightarrow \infty} \int_{\mathbb{R}^{3}} G_{\delta, n}(x) d x=0 .
$$

Consequently,

$$
\begin{aligned}
& \left.\limsup _{n \rightarrow \infty} \int_{\mathbb{R}^{3}}|K|^{6 /(3+2 t)}|| u_{n}\right|^{q}-\left.|u|^{q}\right|^{6 /(3+2 t)} d x \\
& \leq \limsup _{n \rightarrow \infty}\left[\int_{\mathbb{R}^{3}} G_{\delta, n}(x) d x+\delta \int_{\mathbb{R}^{3}}\left(\left|u_{n}\right|^{\frac{6 q r}{r(3+2 t)-6}}-|u|^{\frac{6 q r}{r(3+2 t)-6}}\right) d x\right] \leq C \delta .
\end{aligned}
$$

This shows that

$$
\left.\limsup _{n \rightarrow \infty} \int_{\mathbb{R}^{3}}|K|^{6 /(3+2 t)}|| u_{n}\right|^{q}-\left.|u|^{q}\right|^{6 /(3+2 t)} d x=0,
$$

and then claim (2.10) holds. Similarly, we can deduce that

$$
\lim _{n \rightarrow \infty} \int_{\mathbb{R}^{3}} K(x) \phi_{u}^{t}\left(\left|u_{n}\right|^{q}-|u|^{q}\right) d x=0 .
$$

Notice that

$$
\begin{aligned}
\int_{\mathbb{R}^{3}} K(x)\left(\phi_{u_{n}}^{t}-\phi_{u}^{t}\right)|u|^{q} d x & =\int_{\mathbb{R}^{3}} \int_{\mathbb{R}^{3}} \frac{K(x) K(y)\left|u_{n}(x)\right|^{q}\left(\left|u_{n}(y)\right|^{q}-|u(y)|^{q}\right)}{|x-y|^{3-2 t}} d x d y \\
& =\int_{\mathbb{R}^{3}} K(x) \phi_{u}^{t}\left(\left|u_{n}(x)\right|^{q}-|u(x)|^{q}\right) d x .
\end{aligned}
$$

So, it follow from (2.11)-(2.12) that

$$
\begin{aligned}
& \lim _{n \rightarrow \infty} \int_{\mathbb{R}^{3}} K(x)\left[\phi_{u_{n}}^{t}|u|_{n}^{q}-\phi_{u}^{t}|u|^{q}\right] d x \\
& =\lim _{n \rightarrow \infty}\left[\int_{\mathbb{R}^{3}} K(x) \phi_{u_{n}}^{t}\left(\left|u_{n}\right|^{q}-|u|^{q}\right) d x-\int_{\mathbb{R}^{3}} K(x)\left(\phi_{u_{n}}^{t}-\phi_{u}^{t}\right)|u|^{q} d x\right]=0 .
\end{aligned}
$$

The proof of the second equality in item (ii) is similar, so we omit it and this completes the proof of Proposition 2.4 .

Lemma 2.5. Assume that $(K)$ holds, $(V, h) \in \mathcal{H}$ and $f$ satisfies either $\left(f_{1}\right)$ or $\left(\widetilde{f}_{1}\right)$ and $\left(f_{2}\right)-\left(f_{4}\right)$. For each $u \in H \backslash\{0\}$, there exists a unique $\alpha_{u}^{\prime}>0$ such that $\alpha_{u}^{\prime} u \in \mathcal{N}$. Moreover, $I\left(\alpha_{u}^{\prime} u\right)=\max _{\alpha \geq 0} I(\alpha u)$.

Proof. Firstly, assume that $\left(V h_{2}\right)$ holds. Then, by $\left(f_{1}\right)$ and $\left(f_{4}\right)$, for any $\varepsilon>0$, there exists $C_{\varepsilon}$ such that $|F(u)| \leq$ $\varepsilon|u|^{2}+C_{\varepsilon}|u|^{v}, \quad \forall u \in \mathbb{R}$. Hence, using the Sobolev embedding inequality we get

$$
\begin{aligned}
I(\alpha u)= & \frac{\alpha^{2}}{2} \int_{\mathbb{R}^{3}}\left|(-\Delta)^{\frac{s}{2}} u\right|^{2}+V(x) u^{2} d x+\frac{\alpha^{2 q}}{2 q} \int_{\mathbb{R}^{3}} K(x) \phi_{u}^{t}|u|^{q} d x-\int_{\mathbb{R}^{3}} h(x) F(\alpha u) d x \\
& -\frac{\alpha^{2_{s}^{\star}}}{2_{s}^{\star}} \int_{\mathbb{R}^{3}}|u|^{2_{s}^{\star}} d x \\
\geq & \frac{\alpha^{2}}{2}\|u\|^{2}-\varepsilon \int_{\mathbb{R}^{3}} h(x) \alpha^{2}|u|^{2} d x-C_{\varepsilon} \int_{\mathbb{R}^{3}} h(x) \alpha^{v}|u|^{v} d x-\frac{\alpha^{2_{s}^{\star}}}{2_{s}^{\star}} \int_{\mathbb{R}^{3}}|u|^{2_{s}^{\star}} d x \\
\geq & \alpha^{2}\left(\frac{1}{2}-\varepsilon\left\|h V^{-1}\right\|_{\infty}\right)\|u\|^{2}-\alpha^{v} C C_{\varepsilon}\|h\|_{\infty}\|u\|^{v}-\alpha^{2_{s}^{\star}} C\|u\|^{2_{s}^{\star}} .
\end{aligned}
$$


We can choose $\varepsilon<\frac{1}{2}\left\|h V^{-1}\right\|_{\infty}$ and $\alpha_{0}>0$ sufficiently small such that

$$
h_{u}(\alpha)=I(\alpha u)>0 \text {, for all } \alpha \in\left(0, \alpha_{0}\right) .
$$

Now, we assume that $\left(V h_{3}\right)$ holds. Then, from [9], there exists a positive constant $C_{p}$ such that, for each $\varepsilon \in\left(0, C_{p}\right)$, we obtain $R>0$ such that for each $u \in H$,

$$
\int_{B_{R}^{c}} h(x)|u|^{p} d x \leq \varepsilon \int_{B_{R}^{c}}\left[V(x)|u|^{2}+|u|^{2_{s}^{*}}\right] d x .
$$

By $\left(\widetilde{f}_{1}\right),\left(f_{4}\right),(2.15)$ and the Sobolev embedding inequality, we infer that

$$
\begin{aligned}
I(\alpha u)= & \frac{\alpha^{2}}{2} \int_{\mathbb{R}^{3}}\left|(-\Delta)^{\frac{s}{2}} u\right|^{2}+V(x) u^{2} d x+\frac{\alpha^{2 q}}{2 q} \int_{\mathbb{R}^{3}} K(x) \phi_{u}^{t}|u|^{q} d x-\int_{\mathbb{R}^{3}} h(x) F(\alpha u) d x \\
& -\frac{\alpha^{2_{s}^{*}}}{2_{s}^{\star}} \int_{\mathbb{R}^{3}}|u|^{2_{s}^{*}} d x \\
\geq & \frac{\alpha^{2}}{2}\|u\|^{2}-C \alpha^{p} \int_{\mathbb{R}^{3}} h(x)|u|^{p} d x-C \alpha^{v} \int_{\mathbb{R}^{3}} h(x)|u|^{v} d x-\frac{\alpha^{2_{s}^{*}}}{2_{s}^{\star}} \int_{\mathbb{R}^{3}}|u|^{2_{s}^{*}} d x \\
\geq & \frac{\alpha^{2}}{2}\|u\|^{2}-\varepsilon C \alpha^{p} \int_{B_{R}^{c}}\left[V(x)|u|^{2}+|u|^{2_{s}^{*}}\right] d x-C \alpha^{p} \int_{B_{R}} h(x)|u|^{p} d x \\
& \quad-C \alpha^{v}\|h\|_{\infty} \int_{\mathbb{R}^{3}}|u|^{v} d x-\frac{\alpha^{2_{s}^{*}}}{2_{s}^{\star}} \int_{\mathbb{R}^{3}}|u|^{2_{s}^{*}} d x \\
\geq & \frac{\alpha^{2}}{2}\|u\|^{2}-\varepsilon C \alpha^{p} \int_{B_{R}^{c}}\left[V(x)|u|^{2}+|u|^{2_{s}^{*}}\right] d x-C \alpha^{p}\|h\|_{L^{\infty}\left(B_{R}\right)} \int_{B_{R}}|u|^{p} d x \\
- & C \alpha^{v}\|h\|_{\infty} \int_{\mathbb{R}^{3}}|u|^{v} d x-\frac{\alpha^{2_{s}^{*}}}{2_{s}^{\star}} \int_{\mathbb{R}^{3}}|u|^{2_{s}^{*}} d x \\
\geq & \alpha^{2}\left(\frac{1}{2}-\varepsilon \alpha^{p-2} C_{1}\left\|h V^{-1}\right\| \|_{\infty}\right)\|u\|^{2}-\left(\varepsilon \alpha^{p} C\|u\|^{2_{s}^{*}}+\alpha^{p} C\|h\|_{\infty}\|u\|^{p}\right)-C \alpha^{v}\|h\|_{\infty}\|u\|^{v} \\
- & C \alpha^{2_{s}^{*}}\|u\|^{2_{s}^{*}>0}
\end{aligned}
$$

if $\varepsilon<\min \left\{C_{p}, \frac{1}{2} \alpha^{p-2} C_{1}\left\|h V^{-1}\right\|_{\infty}\right\}$ and $\alpha \in\left(0, \alpha_{0}^{\prime}\right)$ for some small $\alpha_{0}^{\prime}>0$.

On the other hand, since $F(u) \geq 0$ for every $u \in \mathbb{R}$ and $h(x)>0$ for all $x \in \mathbb{R}^{3}$, we have

$$
\begin{aligned}
I(\alpha u) \leq & \frac{\alpha^{2}}{2} \int_{\mathbb{R}^{3}}\left|(-\Delta)^{\frac{s}{2}} u\right|^{2}+V(x) u^{2} d x+\frac{\alpha^{2 q}}{2 q} \int_{\mathbb{R}^{3}} K(x) \phi_{u}^{t}|u|^{q} d x-\int_{A} h(x) F(\alpha u) d x \\
& -\frac{\alpha^{2_{s}^{*}}}{2_{s}^{\star}} \int_{\mathbb{R}^{3}}|u|^{2_{s}^{\star}} d x,
\end{aligned}
$$

where $A \subset \operatorname{supp} u$ is a measurable set with finite and positive measure. Hence,

$$
\limsup _{\alpha \rightarrow+\infty} \frac{I(\alpha u)}{\alpha^{2 q}} \leq \limsup _{\alpha \rightarrow+\infty}\left(\frac{\|u\|^{2}}{2 \alpha^{2 q-2}}+\frac{1}{2 q} \int_{\mathbb{R}^{3}} K(x) \phi_{u}^{t}|u|^{q} d x\right)-\liminf _{\alpha \rightarrow+\infty} \int_{A} h(x) \frac{F(\alpha u)}{(\alpha u)^{2 q}} u^{2 q} d x .
$$

In light of $\left(f_{2}\right)$ and using Fatou's lemma we can infer that

$$
\limsup _{\alpha \rightarrow+\infty} \frac{I(\alpha u)}{\alpha^{2 q}}<0 .
$$

Therefore, there exists $R>0$ sufficiently large such that $h_{u}(R)=I(R u)<0$.

Since $h_{u}$ is a continuous function and exploiting $\left(f_{2}\right)$, there is $\alpha_{u}^{\prime}>0$ which is global maximum of $h_{u}$ with $\alpha_{u}^{\prime} u \in \mathcal{N}$. Next we prove that $\alpha_{u}^{\prime}$ is the unique critical point of $h_{u}$. Assume by contradiction that there are $\alpha_{1}>\alpha_{2}>0$ critical points of $h_{u}$. Then we have

$$
\frac{\|u\|^{2}}{\alpha_{1}^{2 q-2}}+\int_{\mathbb{R}^{3}} K(x) \phi_{u}^{t}|u|^{q} d x-\int_{\mathbb{R}^{3}} h(x) \frac{f\left(\alpha_{1} u\right)}{\left(\alpha_{1} u\right)^{2 q-1}} u^{2 q} d x-\alpha_{1}^{2_{s}^{*}-2 q} \int_{\mathbb{R}^{3}}|u|^{2_{s}^{*}} d x=0,
$$




$$
\frac{\|u\|^{2}}{\alpha_{2}^{2 q-2}}+\int_{\mathbb{R}^{3}} K(x) \phi_{u}^{t}|u|^{q} d x-\int_{\mathbb{R}^{3}} h(x) \frac{f\left(\alpha_{1} u\right)}{\left(\alpha_{2} u\right)^{2 q-1}} u^{2 q} d x-\alpha_{2}^{2_{s}^{*}-2 q} \int_{\mathbb{R}^{3}}|u|^{2_{s}^{*}} d x=0,
$$

From which, taking into account $\left(f_{3}\right)$, we deduce

$$
\begin{aligned}
0> & \left(\frac{1}{\alpha_{1}^{2 q-2}}-\frac{1}{\alpha_{2}^{2 q-2}}\right)\|u\|^{2}-\int_{\mathbb{R}^{3}} h(x)\left[\frac{f\left(\alpha_{1} u\right)}{\left(\alpha_{1} u\right)^{2 q-1}}-\frac{f\left(\alpha_{2} u\right)}{\left(\alpha_{2} u\right)^{2 q-1}}\right] u^{2 q} d x \\
& -\left(\alpha_{1}^{2_{s}^{*}-2 q}-\alpha_{2}^{2_{s}^{*}-2 q}\right) \int_{\mathbb{R}^{3}}|u|^{2_{s}^{\star}} d x=0,
\end{aligned}
$$

which leads a contradiction.

In a standard way (see [40]), we can prove that I satisfies the following mountain-pass geometrical structure.

Lemma 2.6. Assume that $(K)$ holds, $(V, h) \in \mathcal{H}$ and $f$ satisfies either $\left(f_{1}\right)$ or $\left(\widetilde{f}_{1}\right)$ and $\left(f_{2}\right)-\left(f_{4}\right)$. Then I satisfies the following conditions:

(i) There exists $\alpha, \rho>0$ such that $I(u) \geq \alpha$ if $\|u\|=\rho$;

(ii) There exists an $e \in H$ with $\|e\| \geq \rho$ such that $I(e)<0$.

Lemma 2.7. Assume that $(K)$ holds, $(V, h) \in \mathcal{H}$ and $f$ satisfies either $\left(f_{1}\right)$ or $\left(\widetilde{f}_{1}\right)$ and $\left(f_{2}\right)-\left(f_{4}\right)$. Then, we have the following conclusions:

(i) For each $u \in H$ with $u^{ \pm} \neq 0$, there exists a unique pair $\left(\alpha_{u}, \beta_{u}\right) \in \mathbb{R}_{+} \times \mathbb{R}_{+}$such that $\alpha_{u} u^{+}+\beta_{u} u^{-} \in \mathcal{M}$ and $I\left(\alpha_{u} u^{+}+\beta_{u} u^{-}\right)=\max _{\alpha, \beta \geq 0} I\left(\alpha u^{+}+\beta u^{-}\right)$;

(ii) If $\left\langle I^{\prime}(u), u^{ \pm}\right\rangle \leq 0$, then $\left(\alpha_{u}, \beta_{u}\right) \in(0,1] \times(0,1]$;

(iii) $\alpha_{u}, \beta_{u}$ are continuous functional in $H$ with respect to $u$;

(iv) If $u_{n}^{+} \rightarrow 0$, then $\alpha_{u_{n}} \rightarrow \infty$ as $n \rightarrow \infty$; If $u_{n}^{-} \rightarrow 0$, then $\beta_{u_{n}} \rightarrow \infty$ as $n \rightarrow \infty$.

Proof. (i) For each $u \in H$ with $u^{ \pm} \neq 0$. We firstly establish the existence of $\alpha_{u}$ and $\beta_{u}$. Let

$$
\begin{aligned}
g_{1}(\alpha, \beta)= & \left\langle I^{\prime}\left(\alpha u^{+}+\beta u^{-}\right), \alpha u^{+}\right\rangle \\
= & \alpha^{2}\left\|u^{+}\right\|^{2}+\alpha \beta \int_{\mathbb{R}^{3}}(-\Delta)^{\frac{s}{2}} u^{+}(-\Delta)^{\frac{s}{2}} u^{-} d x+\alpha^{2 q} \int_{\mathbb{R}^{3}} K(x) \phi_{u^{+}}^{t}\left|u^{+}\right|^{q} d x \\
& +\alpha^{q} \beta^{q} \int_{\mathbb{R}^{3}} K(x) \phi_{u^{+}}^{t}\left|u^{-}\right|^{q} d x-\int_{\mathbb{R}^{3}} h(x) f\left(\alpha u^{+}\right) \alpha u^{+} d x-\alpha^{2_{s}^{*}} \int_{\mathbb{R}^{3}}\left|u^{+}\right|^{2_{s}^{*}} d x \\
g_{2}(\alpha, \beta)= & \left\langle I^{\prime}\left(\alpha u^{+}+\beta u^{-}\right), \beta u^{-}\right\rangle \\
= & \beta^{2}\left\|u^{-}\right\|^{2}+\alpha \beta \int_{\mathbb{R}^{3}}(-\Delta)^{\frac{s}{2}} u^{+}(-\Delta)^{\frac{s}{2}} u^{-} d x+\beta^{2 q} \int_{\mathbb{R}^{3}} K(x) \phi_{u^{-}}^{t}\left|u^{-}\right|^{q} d x \\
& +\alpha^{q} \beta^{q} \int_{\mathbb{R}^{3}} K(x) \phi_{u^{+}}^{t}\left|u^{-}\right|^{q} d x-\int_{\mathbb{R}^{3}} h(x) f\left(\beta u^{-}\right) \beta u^{-} d x-\beta^{2_{s}^{*}} \int_{\mathbb{R}^{3}}\left|u^{-}\right|^{2_{s}^{*}} d x
\end{aligned}
$$

It is easy to see that $g_{1}(\alpha, \alpha)>0$ and $g_{2}(\alpha, \alpha)>0$ for $\alpha>0$ sufficiently small and $g_{1}(\beta, \beta)<0$ and $g_{2}(\beta, \beta)<0$ for $\beta>0$ sufficiently large. Thus, there exist $r \in(0, R)$ such that

$$
g_{1}(r, \beta)>0, \quad g_{1}(R, \beta)<0, \forall \beta \in[r, R] .
$$

and

$$
g_{2}(\alpha, r)>0, g_{2}(\alpha, R)<0, \quad \forall \alpha \in[r, R] .
$$

By the Miranda's Theorem[20] , there exists some point $\left(\alpha_{u}, \beta_{u}\right) \in(r, R) \times(r, R)$ such that $g_{1}\left(\alpha_{u}, \beta_{u}\right)=$ $g_{2}\left(\alpha_{u}, \beta_{u}\right)=0$. So $\alpha_{u} u^{+}+\beta_{u} u^{-} \in \mathcal{M}$.

Now, we prove the uniqueness of the pair $\left(\alpha_{u}, \beta_{u}\right)$ and consider two cases.

Case $1 u \in \mathcal{M}$. In this case, we have

$$
\left\langle I^{\prime}(u), u^{+}\right\rangle=\left\langle I^{\prime}(u), u^{-}\right\rangle=0
$$


that is

$$
\begin{aligned}
& \left\|u^{+}\right\|^{2}+\int_{\mathbb{R}^{3}}(-\Delta)^{\frac{s}{2}} u^{+}(-\Delta)^{\frac{s}{2}} u^{-} d x+\int_{\mathbb{R}^{3}} K(x) \phi_{u^{+}}^{t}\left|u^{+}\right|^{q} d x+\int_{\mathbb{R}^{3}} K(x) \phi_{u^{+}}^{t}\left|u^{-}\right|^{q} d x \\
= & \int_{\mathbb{R}^{3}} h(x) f\left(u^{+}\right) u^{+} d x+\int_{\mathbb{R}^{3}}\left|u^{+}\right|^{2_{s}^{*}} d x
\end{aligned}
$$

and

$$
\begin{aligned}
& \left\|u^{-}\right\|^{2}+\int_{\mathbb{R}^{3}}(-\Delta)^{\frac{s}{2}} u^{+}(-\Delta)^{\frac{s}{2}} u^{-} d x+\int_{\mathbb{R}^{3}} K(x) \phi_{u^{-}}^{t}\left|u^{-}\right|^{q} d x+\int_{\mathbb{R}^{3}} K(x) \phi_{u^{+}}^{t}\left|u^{-}\right|^{q} d x \\
= & \int_{\mathbb{R}^{3}} h(x) f\left(u^{-}\right) u^{-} d x+\int_{\mathbb{R}^{3}}\left|u^{-}\right|^{2_{s}^{*}} d x .
\end{aligned}
$$

We show that $\left(\alpha_{u}, \beta_{u}\right)=(1,1)$ is the unique pair of numbers such that $\alpha_{u} u^{+}+\beta_{u} u^{-} \in \mathcal{M}$. Suppose that $(\gamma, \eta)$ is another pair of numbers such that $\gamma u^{+}+\eta u^{-} \in \mathcal{M}$. By the definition of $\mathcal{N}$, we have that

$$
\begin{aligned}
& \gamma^{2}\left\|u^{+}\right\|^{2}+\gamma \eta \int_{\mathbb{R}^{3}}(-\Delta)^{\frac{s}{2}} u^{+}(-\Delta)^{\frac{s}{2}} u^{-} d x+\gamma^{2 q} \int_{\mathbb{R}^{3}} K(x) \phi_{u^{+}}^{t}\left|u^{+}\right|^{q} d x \\
& +\gamma^{q} \eta^{q} \int_{\mathbb{R}^{3}} K(x) \phi_{u^{+}}^{t}\left|u^{-}\right|^{q} d x=\int_{\mathbb{R}^{3}} h(x) f\left(\gamma u^{+}\right) \gamma u^{+} d x+\gamma^{2^{\star}} \int_{\mathbb{R}^{3}}\left|u^{+}\right|^{2^{\star}} d x
\end{aligned}
$$

and

$$
\begin{aligned}
& \eta^{2}\left\|u^{-}\right\|^{2}+\gamma \eta \int_{\mathbb{R}^{3}}(-\Delta)^{\frac{s}{2}} u^{+}(-\Delta)^{\frac{s}{2}} u^{-} d x+\eta^{2 q} \int_{\mathbb{R}^{3}} K(x) \phi_{u^{-}}^{t}\left|u^{-}\right|^{q} d x \\
& +\gamma^{q} \eta^{q} \int_{\mathbb{R}^{3}} K(x) \phi_{u^{+}}^{t}\left|u^{-}\right|^{q} d x=\int_{\mathbb{R}^{3}} h(x) f\left(\eta u^{-}\right) \eta u^{-} d x+\eta^{2_{s}^{*}} \int_{\mathbb{R}^{3}}\left|u^{-}\right|^{2_{s}^{*}} d x .
\end{aligned}
$$

Without loss of generality, we may assume that $0<\gamma \leq \eta$. Then from (2.22), we get

$$
\begin{aligned}
& \gamma^{2}\left\|u^{+}\right\|^{2}+\gamma^{2} \int_{\mathbb{R}^{3}}(-\Delta)^{\frac{s}{2}} u^{+}(-\Delta)^{\frac{s}{2}} u^{-} d x+\gamma^{2 q} \int_{\mathbb{R}^{3}} K(x) \phi_{u^{+}}^{t}\left|u^{+}\right|^{q} d x \\
& +\gamma^{2 q} \int_{\mathbb{R}^{3}} K(x) \phi_{u^{+}}^{t}\left|u^{-}\right|^{q} d x \leq \int_{\mathbb{R}^{3}} h(x) f\left(\gamma u^{+}\right) \gamma u^{+} d x+\gamma^{2_{s}^{*}} \int_{\mathbb{R}^{3}}\left|u^{+}\right|^{2_{s}^{*}} d x .
\end{aligned}
$$

Then, dividing the above inequality by $\gamma^{2 q}$, we obtain

$$
\begin{aligned}
& \gamma^{2-2 q}\left\|u^{+}\right\|^{2}+\gamma^{2-2 q} \int_{\mathbb{R}^{3}}(-\Delta)^{\frac{s}{2}} u^{+}(-\Delta)^{\frac{s}{2}} u^{-} d x+\int_{\mathbb{R}^{3}} K(x) \phi_{u^{+}}^{t}\left|u^{+}\right|^{q} d x \\
& +\int_{\mathbb{R}^{3}} K(x) \phi_{u^{+}}^{t}\left|u^{-}\right|^{q} d x \leq \int_{\mathbb{R}^{3}} h(x) \frac{f\left(\gamma u^{+}\right)}{\gamma^{2 q-1}} u^{+} d x+\gamma^{2_{s}^{*}-2 q} \int_{\mathbb{R}^{3}}\left|u^{+}\right|^{2_{s}^{*}} d x .
\end{aligned}
$$

Combining (2.24) with (2.25), we have

$$
\begin{aligned}
& \left(\gamma^{2-2 q}-1\right)\left(\left\|u^{+}\right\|^{2}+\int_{\mathbb{R}^{3}}(-\Delta)^{\frac{s}{2}} u^{+}(-\Delta)^{\frac{s}{2}} u^{-} d x\right) \\
\leq & \int_{\mathbb{R}^{3}} h(x)\left(\frac{f\left(\gamma u^{+}\right)}{\left(\gamma u^{+}\right)^{2 q-1}}-\frac{f\left(u^{+}\right)}{\left(u^{+}\right)^{2 q-1}}\right)\left(u^{+}\right)^{2 q} d x+\left(\gamma^{2_{s}^{*}-2 q}-1\right) \int_{\mathbb{R}^{3}}\left|u^{+}\right|^{2_{s}^{*}} d x .
\end{aligned}
$$

By $\left(f_{3}\right)$ and (2.26), we get $1 \leq \gamma \leq \eta$. Similarly, from (2.21) and (2.23), we have

$$
\begin{aligned}
& \left(\eta^{2-2 q}-1\right)\left(\left\|u^{-}\right\|^{2}+\int_{\mathbb{R}^{3}}(-\Delta)^{\frac{s}{2}} u^{+}(-\Delta)^{\frac{s}{2}} u^{-} d x\right) \\
\geq & \int_{\mathbb{R}^{3}} h(x)\left(\frac{f\left(\eta u^{-}\right)}{\left(\eta u^{-}\right)^{2 q-1}}-\frac{f\left(u^{-}\right)}{\left(u^{-}\right)^{2 q-1}}\right)\left(u^{-}\right)^{2 q} d x+\left(\eta^{2_{s}^{*}-2 q}-1\right) \int_{\mathbb{R}^{3}}\left|u^{-}\right|^{2_{s}^{*}} d x .
\end{aligned}
$$

which implies that $\eta \leq 1$. Together with $1 \leq \gamma \leq \eta$, we show that $\gamma=\eta=1$.

Case $2 u \notin \mathcal{M}$. In this case, there exists $\left(\alpha_{u}, \beta_{u}\right) \in \mathbb{R}_{+} \times \mathbb{R}_{+}$such that $\alpha_{u} u^{+}+\beta_{u} u^{-} \in \mathcal{M}$. Assume that there exists another pair $\left(\gamma_{u}, \eta_{u}\right) \in \mathbb{R}_{+} \times \mathbb{R}_{+}$such that $\gamma_{u} u^{+}+\eta_{u} u^{-} \in \mathcal{M}$. Set $v:=\alpha_{u} u^{+}+\beta_{u} u^{-}$and $v^{\prime}:=\gamma_{u} u^{+}+\eta_{u} u^{-}$, we have

$$
\frac{\gamma_{u}}{\alpha_{u}} v^{+}+\frac{\eta_{u}}{\beta_{u}} v^{-}=\gamma_{u} u^{+}+\eta_{u} u^{-}=v^{\prime} \in \mathcal{M} .
$$


Since $v \in \mathcal{M}$, we obtain that $\alpha_{u}=\gamma_{u}$ and $\beta_{u}=\eta_{u}$. It implies that $\alpha_{u}=\gamma_{u}$ is the unique pair of numbers such that $\alpha_{u} u^{+}+\beta_{u} u^{-} \in \mathcal{M}$.

Define $\omega: \mathbb{R}_{+} \times \mathbb{R}_{+} \rightarrow \mathbb{R}$ by $\omega(\alpha, \beta)=I\left(\alpha u^{+}+\beta u^{-}\right)$. Then we know that $\left(\alpha_{u}, \beta_{u}\right)$ is the unique critical point of $\omega$ in $\mathbb{R}_{+} \times \mathbb{R}_{+}$. It is not difficult to see that $\omega(\alpha, \beta) \rightarrow-\infty$ uniformly as $|(\alpha, \beta)| \rightarrow \infty$, so it is sufficient to show that the maximum point cannot be achieved on the boundary of $\left(\mathbb{R}_{+}, \mathbb{R}_{+}\right)$. Assume that $\left(0, \beta^{\prime}\right)$ is the maximum point of $\omega$ with $\beta^{\prime} \geq 0$. Then, since

$$
\begin{aligned}
\omega\left(\alpha, \beta^{\prime}\right)= & I\left(\alpha u^{+}+\beta^{\prime} u^{-}\right) \\
= & \frac{\alpha^{2}}{2}\left\|u^{+}\right\|^{2}+\frac{\left(\beta^{\prime}\right)^{2}}{2}\left\|u^{-}\right\|^{2}+\alpha \beta^{\prime} \int_{\mathbb{R}^{3}}(-\Delta)^{\frac{s}{2}} u^{+}(-\Delta)^{\frac{s}{2}} u^{-} d x+\frac{\alpha^{2 q}}{2 q} \int_{\mathbb{R}^{3}} K(x) \phi_{u^{+}}^{t}\left|u^{+}\right|^{q} d x \\
& +\frac{\left(\beta^{\prime}\right)^{2 q}}{2 q} \int_{\mathbb{R}^{3}} K(x) \phi_{u^{-}}^{t}\left|u^{-}\right|^{q} d x+\frac{\alpha^{q}\left(\beta^{\prime}\right)^{q}}{2} \int_{\mathbb{R}^{3}} K(x) \phi_{u^{+}}^{t}\left|u^{-}\right|^{q} d x-\int_{\mathbb{R}^{3}} h(x) F\left(\alpha u^{+}\right) d x \\
& -\int_{\mathbb{R}^{3}} h(x) F\left(\beta^{\prime} u^{-}\right) d x-\frac{\alpha^{2_{s}^{*}}}{2_{s}^{\star}} \int_{\mathbb{R}^{3}}\left|u^{+}\right|^{2_{s}^{\star}} d x-\frac{\left(\beta^{\prime}\right)^{2_{s}^{*}}}{2_{s}^{\star}} \int_{\mathbb{R}^{3}}\left|u^{-}\right|^{2_{s}^{\star}} d x
\end{aligned}
$$

is an increasing function with respect to $\alpha$ if $\alpha$ is sufficiently small, the pair $\left(0, \beta^{\prime}\right)$ is not the maximum point of $\omega$ in $\mathbb{R}_{+} \times \mathbb{R}_{+}$.

(ii) From $\left\langle I^{\prime}(u), u^{+}\right\rangle \leq 0$, it deduces that

$$
\begin{aligned}
& \left\|u^{+}\right\|^{2}+\int_{\mathbb{R}^{3}}(-\Delta)^{\frac{s}{2}} u^{+}(-\Delta)^{\frac{s}{2}} u^{-} d x+\int_{\mathbb{R}^{3}} K(x) \phi_{u^{+}}^{t}\left|u^{+}\right|^{q} d x+\int_{\mathbb{R}^{3}} K(x) \phi_{u^{+}}^{t}\left|u^{-}\right|^{q} d x \\
\leq & \int_{\mathbb{R}^{3}} h(x) f\left(u^{+}\right) u^{+} d x+\int_{\mathbb{R}^{3}}\left|u^{+}\right|^{2_{s}^{*}} d x .
\end{aligned}
$$

Since $\alpha_{u} u^{+}+\beta_{u} u^{-} \in \mathcal{M}$, without loss of generality, we suppose that $\alpha_{u} \geq \beta_{u}>0$. Then, we have

$$
\begin{aligned}
& \alpha_{u}^{2}\left\|u^{+}\right\|^{2}+\alpha_{u}^{2} \int_{\mathbb{R}^{3}}(-\Delta)^{\frac{s}{2}} u^{+}(-\Delta)^{\frac{s}{2}} u^{-} d x+\alpha_{u}^{2 q} \int_{\mathbb{R}^{3}} K(x) \phi_{u^{+}}^{t}\left|u^{+}\right|^{q} d x \\
& +\alpha_{u}^{2 q} \int_{\mathbb{R}^{3}} K(x) \phi_{u^{+}}^{t}\left|u^{-}\right|^{q} d x \geq \int_{\mathbb{R}^{3}} h(x) f\left(\alpha_{u} u^{+}\right) \alpha_{u} u^{+} d x+\alpha_{u}^{2_{s}^{*}} \int_{\mathbb{R}^{3}}\left|u^{+}\right|^{2_{s}^{*}} d x .
\end{aligned}
$$

Combining (2.28) with (2.29), we obtain

$$
\begin{aligned}
& \left(1-\alpha_{u}^{2-2 q}\right)\left(\left\|u^{+}\right\|^{2}+\int_{\mathbb{R}^{3}}(-\Delta)^{\frac{s}{2}} u^{+}(-\Delta)^{\frac{s}{2}} u^{-} d x\right) \\
& \leq \int_{\mathbb{R}^{3}} h(x)\left(\frac{f\left(u^{+}\right)}{\left(u^{+}\right)^{2 q-1}}-\frac{f\left(\alpha_{u} u^{+}\right)}{\left(\alpha_{u} u^{+}\right)^{2 q-1}}\right)\left(u^{+}\right)^{2 q} d x+\left(1-\alpha^{2_{s}^{*}-2 q}\right) \int_{\mathbb{R}^{3}}\left|u^{+}\right|^{2_{s}^{*}} d x .
\end{aligned}
$$

If $\alpha_{u} \geq 1$, then the left side of this inequality is positive and the right side of this inequality is always negative by $\left(f_{3}\right)$. It implies that $\alpha_{u} \leq 1$ and thus $0<\beta_{u} \leq \alpha_{u} \leq 1$.

(iii) Taking a sequence $\left\{u_{n}\right\} \subset H$ such that $u_{n} \rightarrow u$ in $H$, then $u_{n}^{ \pm} \rightarrow u^{ \pm}$in $H$. By item (i), there exist $\left(\alpha_{u_{n}}, \beta_{u_{n}}\right)$ and $\left(\alpha_{u}, \beta_{u}\right)$ such that $\alpha_{u_{n}} u_{n}^{+}+\beta_{u_{n}} u_{n}^{-} \in \mathcal{M}$ and $\alpha_{u} u^{+}+\beta_{u} u^{-} \in \mathcal{M}$. Then

$$
\begin{aligned}
& \alpha_{u_{n}}^{2}\left\|u_{n}^{+}\right\|^{2}+\alpha_{u_{n}} \beta u_{n} \int_{\mathbb{R}^{3}}(-\Delta)^{\frac{s}{2}} u_{n}^{+}(-\Delta)^{\frac{s}{2}} u_{n}^{-} d x+\alpha_{u_{n}}^{2 q} \int_{\mathbb{R}^{3}} K(x) \phi_{u_{n}^{+}}^{t}\left|u_{n}^{+}\right|^{q} d x \\
& +\alpha_{u_{n}}^{q} \beta_{u_{n}}^{q} \int_{\mathbb{R}^{3}} K(x) \phi_{u_{n}^{+}}^{t}\left|u_{n}^{-}\right|^{q} d x=\int_{\mathbb{R}^{3}} h(x) f\left(\alpha_{u_{n}} u_{n}^{+}\right) \alpha_{u_{n}} u_{n}^{+} d x+\alpha_{u_{n}}^{2_{s}^{*}} \int_{\mathbb{R}^{3}}\left|u_{n}^{+}\right|^{2_{s}^{*}} d x
\end{aligned}
$$

and

$$
\begin{aligned}
& \beta_{u_{n}}^{2}\left\|u_{n}^{-}\right\|^{2}+\alpha_{u_{n}} \beta u_{n} \int_{\mathbb{R}^{3}}(-\Delta)^{\frac{s}{2}} u_{n}^{+}(-\Delta)^{\frac{s}{2}} u_{n}^{-} d x+\beta_{u_{n}}^{2 q} \int_{\mathbb{R}^{3}} K(x) \phi_{u_{n}^{-}}^{t}\left|u_{n}^{-}\right|^{q} d x \\
& +\alpha_{u_{n}}^{q} \beta_{u_{n}}^{q} \int_{\mathbb{R}^{3}} K(x) \phi_{u_{n}^{+}}^{t}\left|u_{n}^{-}\right|^{q} d x=\int_{\mathbb{R}^{3}} h(x) f\left(\beta_{u_{n}} u_{n}^{-}\right) \beta_{u_{n}} u_{n}^{-} d x+\beta_{u_{n}}^{2_{s}^{*}} \int_{\mathbb{R}^{3}}\left|u_{n}^{-}\right|^{2_{s}^{*}} d x .
\end{aligned}
$$

It is obvious that $\alpha_{u_{n}}, \beta_{u_{n}} \geq C$ for some constant $C>0$. Next we claim that $\left\{\alpha_{u_{n}}\right\}$ and $\left\{\beta_{u_{n}}\right\}$ are bounded in $\mathbb{R}^{+}$. In fact, without loss of generality, we suppose that $\alpha_{u_{n}} \rightarrow \infty$ as $n \rightarrow \infty$, by $u_{n}^{ \pm} \rightarrow u^{ \pm} \neq 0$ in $H$ and dividing the above (2.31) by $\alpha_{u_{n}}^{2 q}$, we infer that

$$
\frac{\beta_{u_{n}}}{\alpha_{u_{n}}^{2 q-1}} \rightarrow+\infty \text { or } \frac{\beta_{u_{n}}}{\alpha_{u_{n}}} \rightarrow+\infty \text { as } n \rightarrow \infty,
$$


and thus

$$
\beta_{u_{n}} \rightarrow+\infty \text { as } n \rightarrow \infty \text {. }
$$

If $\frac{\beta_{u_{n}}}{\alpha_{u_{n}}^{2-1}} \rightarrow+\infty$ as $n \rightarrow \infty$, then by (2.32), we have

$$
\frac{\alpha_{u_{n}}}{\beta_{u_{n}}^{2 q-1}} \rightarrow+\infty \text { or } \frac{\alpha_{u_{n}}}{\beta u_{n}} \rightarrow+\infty \text { as } n \rightarrow \infty,
$$

which leads a contradiction because

$$
\frac{\alpha_{u_{n}}}{\beta_{u_{n}}^{2 q-1}}=\frac{\alpha_{u_{n}}^{(2 q-1)^{2}}}{\beta_{u_{n}}^{2 q-1}} \cdot \frac{1}{\alpha_{u_{n}}^{(2 q-1)^{2}-1}} \rightarrow 0 \text { or } \frac{\alpha_{u_{n}}}{\beta_{u_{n}}}=\frac{\alpha_{u_{n}}^{2 q-1}}{\beta_{u_{n}}} \cdot \frac{1}{\alpha_{u_{n}}^{2 q-2}} \rightarrow 0 \text { as } n \rightarrow \infty .
$$

Similarly, if $\frac{\beta_{u_{n}}}{\alpha_{u_{n}}} \rightarrow+\infty$ as $n \rightarrow \infty$, we also obtain a contradiction. Therefore, up to a subsequence, still denoted by $\left\{\alpha_{u_{n}}\right\}$ and $\left\{\beta_{u_{n}}\right\}$, we assume that there exists a unique pair $\left(\alpha_{0}, \beta_{0}\right) \in \mathbb{R}_{+} \times \mathbb{R}_{+}$such that

$$
\lim _{n \rightarrow+\infty} \alpha_{u_{n}}=\alpha_{0}>0, \quad \lim _{n \rightarrow+\infty} \beta_{u_{n}}=\beta_{0}>0 .
$$

Letting $n \rightarrow \infty$ in (2.31) and (2.32), we have

$$
\begin{aligned}
& \alpha_{0}^{2}\left\|u^{+}\right\|^{2}+\alpha_{0} \beta_{0} \int_{\mathbb{R}^{3}}(-\Delta)^{\frac{s}{2}} u^{+}(-\Delta)^{\frac{s}{2}} u^{-} d x+\alpha_{0}^{2 q} \int_{\mathbb{R}^{3}} K(x) \phi_{u^{+}}^{t}\left|u^{+}\right|^{q} d x \\
& +\alpha_{0}^{q} \beta_{0}^{q} \int_{\mathbb{R}^{3}} K(x) \phi_{u^{+}}^{t}\left|u^{-}\right|^{q} d x=\int_{\mathbb{R}^{3}} h(x) f\left(\alpha_{0} u^{+}\right) \alpha_{0} u^{+} d x+\alpha_{0}^{2_{s}^{*}} \int_{\mathbb{R}^{3}}\left|u^{+}\right|^{2_{s}^{\star}} d x
\end{aligned}
$$

and

$$
\begin{aligned}
& \beta_{0}^{2}\left\|u^{-}\right\|^{2}+\alpha_{0} \beta_{0} \int_{\mathbb{R}^{3}}(-\Delta)^{\frac{s}{2}} u^{+}(-\Delta)^{\frac{s}{2}} u^{-} d x+\beta_{0}^{2 q} \int_{\mathbb{R}^{3}} K(x) \phi_{u^{-}}^{t}\left|u^{-}\right|^{q} d x \\
& +\alpha_{0}^{q} \beta_{0}^{q} \int_{\mathbb{R}^{3}} K(x) \phi_{u^{+}}^{t}\left|u^{-}\right|^{q} d x=\int_{\mathbb{R}^{3}} h(x) f\left(\beta_{0} u^{-}\right) \beta_{0} u^{-} d x+\beta_{0}^{2_{s}^{*}} \int_{\mathbb{R}^{3}}\left|u^{-}\right|^{2_{s}^{*}} d x
\end{aligned}
$$

which implies that $\alpha_{0} u^{+}+\beta_{0} u^{-} \in \mathcal{M}$. According to the uniqueness of $\left(\alpha_{u}, \beta_{u}\right)$, we deduce $\alpha_{u}=\alpha_{0}$ and $\beta_{u}=\beta_{0}$. Hence, $\alpha_{u}, \beta_{u}$ are continuous functional in $H$ with respect to $u$.

(iv) We argue by contradiction. If $u_{n}^{+} \rightarrow 0$ in $H$ and there exists $M$ such that $\alpha_{u_{n}} \leq M$. By either $\left(f_{1}\right)$ or $\left(\widetilde{f}_{1}\right)$, $\left(f_{4}\right)$ and the Sobolev embedding inequality, we have that

$$
\begin{aligned}
0= & \frac{\left\langle I^{\prime}\left(\alpha_{u_{n}} u_{n}+\beta_{u_{n}} u_{n}{ }^{-}\right), \alpha_{u_{n}} u_{n}{ }^{+}\right\rangle}{\alpha_{u_{n}}^{2}} \\
= & \left\|u_{n}^{+}\right\|^{2}+\frac{\beta_{u_{n}}}{\alpha_{u_{n}}} \int_{\mathbb{R}^{3}}(-\Delta)^{\frac{s}{2}} u_{n}^{+}(-\Delta)^{\frac{s}{2}} u_{n}^{-} d x+\alpha_{u_{n}}^{2 q-2} \int_{\mathbb{R}^{3}} K(x) \phi_{u_{n}^{+}}^{t}\left|u_{n}^{+}\right|^{q} d x \\
& +\alpha_{n}^{q-2} \beta_{u_{n}}^{q} \int_{\mathbb{R}^{3}} K(x) \phi_{u_{n}^{+}}^{t}\left|u_{n}^{-}\right|^{q} d x-\int_{\mathbb{R}^{3}} h(x) \frac{f\left(\alpha_{u_{n}} u_{n}^{+}\right)}{\alpha_{u_{n}}} u_{n}^{+} d x-\alpha_{u_{n}}^{2_{s}^{*}-2} \int_{\mathbb{R}^{3}}\left|u_{n}^{+}\right|^{2_{s}^{*}} d x \\
\geq & \left\|u_{n}^{+}\right\|^{2}-\|h\|_{\infty}\left(C_{1} M^{2 q-2}\left\|u_{n}^{+}\right\|_{2 q}^{2 q}+C_{2} M^{p-2} \mid u_{n}^{+}\left\|_{p}^{p}+C_{3} M^{v-2}\right\| u_{n}^{+} \|_{v}^{v}\right)-M^{2_{s}^{*}-2}\left\|u_{n}^{+}\right\|_{2_{s}^{*}}^{2_{s}^{*}} \\
\geq & \left\|u_{n}^{+}\right\|^{2}-C\left\|u_{n}^{+}\right\|^{2 q}-C\left\|u_{n}^{+}\right\|^{p}-C\left\|u_{n}^{+}\right\|^{v}-C\left\|u_{n}^{+}\right\|^{2_{s}^{*}}>0,
\end{aligned}
$$

as $n$ large enough, which contradicts the fact that $\alpha_{u_{n}} u_{n}^{+}+\beta_{u_{n}} u_{n}^{-} \in \mathcal{M}$. Hence $\alpha_{u_{n}} \rightarrow \infty$. Similarly, we can prove that $\beta_{u_{n}} \rightarrow \infty$ if $u_{n}^{-} \rightarrow 0$ in $H$.

\section{Existence of positive solution for (1.1)}

In this section, we are going to show the existence of positive solution for problem (1.1), and prove Theorem 1.1. We first present the following lemma implies that $I$ satisfies the local $(P S)_{c}$-condition. 
Lemma 3.1. Assume that $(K)$ holds, $(V, h) \in \mathcal{H}$ and $f$ satisfies either $\left(f_{1}\right)$ or $\left(\widetilde{f}_{1}\right)$ and $\left(f_{2}\right)-\left(f_{4}\right)$. Then, I satisfies the $(P S)_{c}$-condition for $c \in\left(0, \frac{s}{3} \mathcal{S}_{S}^{\frac{3}{2 s}}\right)$.

Proof. Let $\left\{u_{n}\right\}$ be a $(P S)_{c}$-sequence of $I$ at the level $c \in\left(0, \frac{s}{3} S_{s}^{\frac{3}{2 s}}\right)$, that is

$$
I\left(u_{n}\right) \rightarrow c \text { and } I^{\prime}\left(u_{n}\right) \rightarrow 0 \text { in } H^{-1} .
$$

Then, by $\left(f_{3}\right)$ and $h(x)>0$ in $\mathbb{R}^{3}$, we get

$$
\begin{aligned}
& c+1+\left\|u_{n}\right\| \\
& \geq I\left(u_{n}\right)-\frac{1}{2 q}\left\langle I^{\prime}\left(u_{n}\right), u_{n}\right\rangle \\
& =\frac{q-1}{2 q}\left\|u_{n}\right\|^{2}+\frac{1}{2 q} \int_{\mathbb{R}^{3}} h(x)\left(u_{n} f\left(u_{n}\right)-2 q F\left(u_{n}\right)\right) d x+\left(\frac{1}{2 q}-\frac{1}{2_{s}^{*}}\right) \int_{\mathbb{R}^{3}}\left|u_{n}\right|^{2_{s}^{*}} d x \\
& \geq \frac{q-1}{2 q}\left\|u_{n}\right\|^{2},
\end{aligned}
$$

which implies $\left\{u_{n}\right\}$ is bounded in $H$. Thus, there exists a subsequence of $\left\{u_{n}\right\}$, still denoted by $\left\{u_{n}\right\}$ such that

$$
\left\{\begin{array}{l}
u_{n} \rightarrow u \text { in } H, \\
u_{n} \rightarrow u \text { in } L_{l o c}^{r}\left(\mathbb{R}^{3}\right) \text { for } r \in\left[1,2_{s}^{\star}\right), \\
u_{n}(x) \rightarrow u(x) \text { a.e. in } \mathbb{R}^{3} .
\end{array}\right.
$$

From (3.1) and (3.2), it is easy to check that $u$ is a critical point of $I$. Therefore, $\left\langle I^{\prime}(u), u\right\rangle=0$. Then, by $h(x)>$ $0,\left(f_{3}\right)$ and $1<q<2{ }_{s}^{*} / 2$, we have that

$$
\begin{aligned}
I(u) & =I(u)-\frac{1}{2 q}\left\langle I^{\prime}(u), u\right\rangle \\
& =\frac{q-1}{2 q}\|u\|^{2}+\frac{1}{2 q} \int_{\mathbb{R}^{3}} h(x)(u f(u)-2 q F(u)) d x+\left(\frac{1}{2 q}-\frac{1}{2_{s}^{\star}}\right) \int_{\mathbb{R}^{3}}|u|^{2_{s}^{*}} d x \\
& \geq \frac{q-1}{2 q}\|u\|^{2} \geq 0 .
\end{aligned}
$$

Let $v_{n}=u_{n}-u$ and so $v_{n} \rightarrow 0$ in $H$. In view of (3.2), Proposition 2.4 (ii)(a) and the Brezis-Lieb Lemma [7], we obtain that

$$
\begin{aligned}
I\left(u_{n}\right)= & \frac{1}{2}\left\|u_{n}\right\|^{2}+\frac{1}{2 q} \int_{\mathbb{R}^{3}} K(x) \phi_{u_{n}}^{t}\left|u_{n}\right|^{q} d x-\int_{\mathbb{R}^{3}} h(x) F\left(u_{n}\right) d x-\frac{1}{2_{s}^{\star}} \int_{\mathbb{R}^{3}}\left|u_{n}\right|^{2_{s}^{\star}} d x \\
= & \frac{1}{2}\left(\left\|v_{n}\right\|^{2}+\|u\|^{2}\right)+\frac{1}{2 q} \int_{\mathbb{R}^{3}} K(x) \phi_{u}^{t}|u|^{q} d x-\int_{\mathbb{R}^{3}} h(x) F(u) d x \\
& -\frac{1}{2_{s}^{\star}} \int_{\mathbb{R}^{3}}\left|v_{n}\right|^{2_{s}^{*}} d x-\frac{1}{2_{s}^{\star}} \int_{\mathbb{R}^{3}}|u|^{2_{s}^{*}} d x+o_{n}(1) \\
= & I(u)+\frac{1}{2}\left\|v_{n}\right\|^{2}-\frac{1}{2_{s}^{\star}} \int_{\mathbb{R}^{3}}\left|v_{n}\right|^{2_{s}^{*}} d x+o_{n}(1) .
\end{aligned}
$$

Similarly, we have that

$$
\left\langle I^{\prime}\left(u_{n}\right), u_{n}\right\rangle=\left\langle I^{\prime}(u), u\right\rangle+\left\|v_{n}\right\|^{2}-\int_{\mathbb{R}^{3}}\left|v_{n}\right|^{2_{s}^{*}} d x+o_{n}(1) .
$$

Therefore, it follows from (3.1) that

$$
c=\lim _{n \rightarrow+\infty} I\left(u_{n}\right)=I(u)+\frac{1}{2} \lim _{n \rightarrow+\infty}\left\|v_{n}\right\|^{2}-\frac{1}{2_{s}^{\star}} \lim _{n \rightarrow+\infty} \int_{\mathbb{R}^{3}}\left|v_{n}\right|^{2_{s}^{\star}} d x
$$

and

$$
\lim _{n \rightarrow+\infty}\left\|v_{n}\right\|^{2}-\lim _{n \rightarrow+\infty} \int_{\mathbb{R}^{3}}\left|v_{n}\right|^{2_{s}^{*}} d x=0 .
$$


Now, we assume that

$$
\lim _{n \rightarrow+\infty}\left\|v_{n}\right\|^{2}=\ell \text { and } \lim _{n \rightarrow+\infty} \int_{\mathbb{R}^{3}}\left|v_{n}\right|^{2_{s}^{*}} d x=\ell .
$$

By (2.1) we have

$$
\left\|v_{n}\right\|^{2} \geq \int_{\mathbb{R}^{3}}\left|(-\Delta)^{\frac{s}{2}} v_{n}\right|^{2} d x \geq \mathcal{S}_{s}\left(\int_{\mathbb{R}^{3}}\left|v_{n}\right|^{2_{s}^{\star}} d x\right)^{\frac{2}{2_{s}^{\star}}},
$$

which implies that

$$
\ell \geq \mathcal{S}_{S} \ell^{\frac{2}{2_{s}^{*}}} .
$$

Thus, we get that $\ell=0$ or $\ell \geq \mathcal{S}_{S}^{\frac{3}{2 s}}$. Suppose that $\ell \geq \mathcal{S}_{S}^{\frac{3}{2 s}}$, then combining (3.4) with (3.5), we conclude that

$$
c \geq \frac{1}{2} \ell-\frac{1}{2_{s}^{\star}} \ell=\frac{s}{3} \ell \geq \frac{s}{3} S_{s}^{\frac{3}{2 s}},
$$

which contradicts the fact that $c<\frac{s}{3} \mathcal{S}_{s}^{\frac{3}{2 s}}$. Hence, $\ell=0$. This completes the proof of Lemma 3.1.

Lemma 3.2. Assume that $(K)$ holds, $(V, h) \in \mathcal{H}$ and $f$ satisfies either $\left(f_{1}\right)$ or $\left(\widetilde{f}_{1}\right)$ and $\left(f_{2}\right)-\left(f_{4}\right)$. Then, $0<c_{0}<$ $\frac{s}{3} \mathcal{S}_{S}^{\frac{3}{2 s}}$, where $c_{0}$ is given in (2.6).

Proof. For any $u \in \mathcal{N}$, we have $\left\langle I^{\prime}(u), u\right\rangle=0$. Then, by either $\left(f_{1}\right)$ or $\left(\widetilde{f}_{1}\right),\left(f_{4}\right)$ and the Sobolev embedding inequality, we have that

$$
\begin{aligned}
\|u\|^{2} & \leq\|u\|^{2}+\int_{\mathbb{R}^{3}} K(x) \phi_{u}^{t}|u|^{q} d x \\
& =\int_{\mathbb{R}^{3}} h(x) f(u) u d x+\int_{\mathbb{R}^{3}}|u|^{2_{s}^{\star}} d x \\
& \leq C\|h\|_{\infty}\left(\int_{\mathbb{R}^{3}}|u|^{2 q} d x+\int_{\mathbb{R}^{3}}|u|^{p} d x+\int_{\mathbb{R}^{3}}|u|^{v} d x\right)+\int_{\mathbb{R}^{3}}|u|^{2^{\star}} d x \\
& \leq C\|u\|^{2 q}+C\|u\|^{p}+C\|u\|^{v}+C\|u\|^{2_{s}^{\star}},
\end{aligned}
$$

which implies that there exists some constant $C>0$ such that $\|u\| \geq C$. Therefore, we have

$$
\begin{aligned}
I(u) & =I(u)-\frac{1}{2 q}\left\langle I^{\prime}(u), u\right\rangle \\
& =\frac{q-1}{2 q}\|u\|^{2}+\frac{1}{2 q} \int_{\mathbb{R}^{3}} h(x)(u f(u)-2 q F(u)) d x+\left(\frac{1}{2 q}-\frac{1}{2_{s}^{\star}}\right) \int_{\mathbb{R}^{3}}|u|^{2_{s}^{\star}} d x \\
& \geq \frac{q-1}{2 q}\|u\|^{2},
\end{aligned}
$$

which implies that $c_{0} \geq \frac{q-1}{2 q} C^{2}>0$.

Now, we prove $c_{0}<\frac{s}{3} \mathcal{S}_{S}^{\frac{3}{2 s}}$. We define (see [26])

$$
u_{\varepsilon}(x)=\psi(x) U_{\varepsilon}(x), \quad x \in \mathbb{R}^{3},
$$

where $U_{\varepsilon}(x)=\varepsilon^{-(3-2 s) / 2} u^{\star}(x / \varepsilon), u^{*}(x)=\widetilde{u}\left(x / \mathcal{S}_{s}^{1 / 2 s}\right) /\|\widetilde{u}\|_{2_{s}^{\star}}$ Let $\kappa \in \mathbb{R} \backslash\{0\}, \mu>0$ and $x_{0} \in \mathbb{R}^{3}$ be fixed constants, $\widetilde{u}(x)=\kappa\left(\mu^{2}+\left|x-x_{0}\right|^{2}\right)^{-(3-2 s) / 2}$, and let $\psi \in \mathcal{C}_{0}^{\infty}\left(\mathbb{R}^{3}\right)$ such that $0 \leq \psi \leq 1$ in $\mathbb{R}^{3}, \psi(x) \equiv 1$ in $B_{r_{0} / 2}$ and $\psi(x) \equiv 0$ in $\mathbb{R}^{3} \backslash B_{r_{0}}$, where $r_{0}$ is given in Remark 1.1. From Proposition 2.1 and Proposition 2.2 in [26], we know that

$$
\begin{gathered}
\int_{\mathbb{R}^{3}}\left|(-\Delta)^{\frac{s}{2}} u_{\varepsilon}\right|^{2} d x \leq \mathcal{S}_{s}^{\frac{3}{2 s}}+O\left(\varepsilon^{3-2 s}\right), \\
\int_{\mathbb{R}^{3}}\left|u_{\varepsilon}\right|^{2^{*}} d x=\mathcal{S}_{S}^{\frac{3}{2 s}}+O\left(\varepsilon^{3}\right) .
\end{gathered}
$$


By a simple computation, we have

$$
\int_{\mathbb{R}^{3}}\left|u_{\varepsilon}\right|^{r} d x= \begin{cases}O\left(\varepsilon^{\frac{3(2-r)+2 s r}{2}}\right), & r>\frac{3}{3-2 s}, \\ O\left(\varepsilon^{\frac{3(2-r)+2 s r}{2}}|\log \varepsilon|\right), & r=\frac{3}{3-2 s}, \\ O\left(\varepsilon^{\frac{(3-2 s) r}{2}}\right), & r<\frac{3}{3-2 s} .\end{cases}
$$

By Lemma 2.5, we know that there exists a unique $\alpha_{\varepsilon}>0$ such that $\alpha_{\varepsilon} u_{\varepsilon} \in \mathcal{N}$. Thus, by the definition of $c_{0}$, we have $c_{0} \leq I\left(\alpha_{\varepsilon} u_{\varepsilon}\right)$. To complete the proof it suffices to prove that

$$
\max _{\alpha \geq 0} I\left(\alpha u_{\varepsilon}\right)<\frac{s}{3} S_{s}^{\frac{3}{2 s}} .
$$

Since $\max _{\alpha \geq 0} I\left(\alpha u_{\varepsilon}\right)=I\left(\alpha_{\varepsilon} u_{\varepsilon}\right) \geq c_{0}>0$, there exists $A_{1}>0$ such that $\alpha_{\varepsilon} \geq A_{1}>0$. Moreover, since $I\left(\alpha u_{\varepsilon}\right) \rightarrow$ $-\infty$ as $\alpha \rightarrow \infty$ and $I\left(\alpha_{\varepsilon} u_{\varepsilon}\right) \geq c_{0}>0$, we get that there exists $A_{2}>0$ such that $\alpha_{\varepsilon} \leq A_{2}$, and so $0<A_{1} \leq \alpha_{\varepsilon} \leq A_{2}$. Notice that

$$
\begin{aligned}
I\left(\alpha_{\varepsilon} u_{\varepsilon}\right)= & \frac{\alpha_{\varepsilon}^{2}}{2} \int_{\mathbb{R}^{3}}\left|(-\Delta)^{\frac{s}{2}} u_{\varepsilon}\right|^{2}+V(x) u_{\varepsilon}^{2} d x+\frac{\alpha_{\varepsilon}^{2 q}}{2 q} \int_{\mathbb{R}^{3}} K(x) \phi_{u_{\varepsilon}}^{t}|u|_{\varepsilon}^{q} d x-\int_{\mathbb{R}^{3}} h(x) F\left(\alpha_{\varepsilon} u_{\varepsilon}\right) d x \\
& -\frac{\alpha_{\varepsilon}^{2_{s}^{*}}}{2_{s}^{\star}} \int_{\mathbb{R}^{3}}\left|u_{\varepsilon}\right|^{2_{s}^{*}} d x \\
& =I_{1}+I_{2}+I_{3}
\end{aligned}
$$

where

$$
\begin{aligned}
& I_{1}=\frac{\alpha_{\varepsilon}^{2}}{2} \int_{\mathbb{R}^{3}}\left|(-\Delta)^{\frac{s}{2}} u_{\varepsilon}\right|^{2} d x-\frac{\alpha_{\varepsilon}^{2_{s}^{*}}}{2_{s}^{*}} \int_{\mathbb{R}^{3}}\left|u_{\varepsilon}\right|^{2_{s}^{*}} d x, \\
& I_{2}=\frac{\alpha_{\varepsilon}^{2}}{2} \int_{\mathbb{R}^{3}} V(x) u_{\varepsilon}^{2} d x-\int_{\mathbb{R}^{3}} h(x) F\left(\alpha_{\varepsilon} u_{\varepsilon}\right) d x, \\
& I_{3}=\frac{\alpha_{\varepsilon}^{2 q}}{2 q} \int_{\mathbb{R}^{3}} K(x) \phi_{u_{\varepsilon}}^{t}\left|u_{\varepsilon}\right|^{q} d x .
\end{aligned}
$$

First, we claim that

$$
I_{1} \leq \frac{s}{3} \mathcal{S}_{s}^{\frac{3}{2 s}}+O\left(\varepsilon^{3-2 s}\right)
$$

In fact, we define

$$
g(\alpha)=\frac{\alpha^{2}}{2} \int_{\mathbb{R}^{3}}\left|(-\Delta)^{\frac{s}{2}} u_{\varepsilon}\right|^{2} d x-\frac{\alpha^{2_{s}^{*}}}{2_{s}^{\star}} \int_{\mathbb{R}^{3}}\left|u_{\varepsilon}\right|^{2_{s}^{\star}} d x, \text { for } \alpha \geq 0 .
$$

It is easy to see that $g(\alpha)$ attains its maximum at

$$
\alpha_{0}=\left(\frac{\int_{\mathbb{R}^{3}}\left|(-\Delta)^{\frac{s}{2}} u_{\varepsilon}\right|^{2} d x}{\int_{\mathbb{R}^{3}}\left|u_{\varepsilon}\right|^{2_{s}^{*}} d x}\right)^{\frac{3-2 s}{4 s}} .
$$

Therefore, by (3.6) and (3.7), we deduce that

$$
\max _{\alpha \geq 0} g(\alpha)=g\left(\alpha_{0}\right)=\frac{s}{3} \frac{\left\|(-\Delta)^{\frac{s}{2}} u_{\varepsilon}\right\|_{2}^{\frac{3}{2 s}}}{\left\|u_{\varepsilon}\right\|_{2_{s}^{2 s}}^{\frac{3-2 s}{2 s}}} \leq \frac{s}{3} \frac{\left(\mathcal{S}_{s}^{\frac{3}{2 s}}+O\left(\varepsilon^{3-2 s}\right)\right)^{\frac{3}{2 s}}}{\left(\mathcal{S}_{s}^{\frac{3}{2 s}}+O\left(\varepsilon^{3}\right)\right)^{\frac{3-2 s}{2 s}}} \leq \frac{s}{3} \delta_{s}^{\frac{3}{2 s}}+O\left(\varepsilon^{3-2 s}\right),
$$

which implies that (3.10) holds. 
From $\left(f_{4}\right), A_{1} \leq \alpha_{\varepsilon} \leq A_{2}$ and $\mu>2 q>\frac{3+2 t}{3-2 s}>\frac{4 s}{3-2 s}>\frac{3}{3-2 s}$, we have by (3.8) and Remark 1.1,

$$
\begin{aligned}
I_{2} & \leq \frac{A_{2}^{2}}{2} \int_{\mathbb{R}^{3}} V(x)\left|u_{\varepsilon}\right|^{2} d x-\int_{\mathbb{R}^{3}} h(x) F\left(\alpha_{\varepsilon} u_{\varepsilon}\right) d x \\
& =\frac{A_{2}^{2}}{2} \int_{B_{r_{0}}} V(x)\left|u_{\varepsilon}\right|^{2} d x-\int_{B_{r_{0}}} h(x) F\left(\alpha_{\varepsilon} u_{\varepsilon}\right) d x \\
& \leq \frac{A_{2}^{2}}{2} \max _{x \in \bar{B}_{r_{0}}} V(x) \int_{B_{r_{0}}}\left|u_{\varepsilon}\right|^{2} d x-\min _{x \in \bar{B}_{r_{0}}} h(x) \int_{B_{r_{0}}} F\left(\alpha_{\varepsilon} u_{\varepsilon}\right) d x \\
& \leq C_{1} \int_{\mathbb{R}^{3}}\left|u_{\varepsilon}\right|^{2} d x-\lambda h_{0} A_{1}^{\mu} \int_{\mathbb{R}^{3}} u_{\varepsilon}^{\mu} d x \\
& =O\left(\varepsilon^{3-2 s}\right)-O\left(\varepsilon^{3-\frac{\mu(3-2 s)}{2}}\right) .
\end{aligned}
$$

By virtue of $\mu>2 q>\frac{4 s}{3-2 s}$, we see that $3-2 s>3-\frac{\mu(3-2 s)}{2}$. Therefore, to finish the proof, it is enough to prove that

$$
\lim _{\varepsilon \rightarrow 0^{+}} \frac{I_{3}}{\varepsilon^{3-\frac{\mu(3-2 s)}{2}}}=0 .
$$

In fact, by $K(x) \in L^{\infty}\left(\mathbb{R}^{3}\right)$ and Proposition 2.2, we have

$$
\int_{\mathbb{R}^{3}} \int_{\mathbb{R}^{3}} \frac{K(x)\left|u_{\varepsilon}(x)\right|^{q} K(y)\left|u_{\varepsilon}(y)\right|^{q}}{|x-y|^{3-2 t}} d y d x \leq C\left(\int_{\mathbb{R}^{3}}\left|u_{\varepsilon}\right|^{\frac{6 q}{3+2 t}} d x\right)^{\frac{3+2 t}{3}} .
$$

Moreover, by $\mu>2 q>\frac{2(3 q-2 s q-2 t)}{3-2 s}, q>\frac{3+2 t}{2(3-2 s)}$ and (3.8), we have that

$$
\lim _{\varepsilon \rightarrow 0^{+}} \frac{\left(\int_{\mathbb{R}^{3}}\left|u_{\varepsilon}\right|^{\frac{6 q}{3+2 t}} d x\right)^{\frac{3+2 t}{3}}}{\varepsilon^{3-\frac{\mu(3-2 s)}{2}}}=\lim _{\varepsilon \rightarrow 0^{+}} \frac{O\left(\varepsilon^{2 s q+2 t+3-3 q}\right)}{\varepsilon^{3-\frac{\mu(3-2 s)}{2}}}=0 .
$$

Hence, (3.13) holds and then we see that (3.9) holds and this completes the proof.

Proof of Theorem 1.1. By Lemma 2.6, Lemma 3.1 and Lemma 3.2, we know that the functional $I$ satisfies the mountain-pass geometrical structure and the $(P S)_{c_{0}}$-condition. Hence, the functional $I$ has a critical value $c_{0}>0$. That is, there exists a non-trivial $u_{0} \in H$ such that $I\left(u_{0}\right)=c_{0}$ and $I^{\prime}\left(u_{0}\right)=0$, which implies that $u_{0}$ is the non-trivial ground state solution of problem (1.1).

Next, we prove that $u_{0}$ is positive. It is easy to see that all the above calculations can be repeated word by word, replacing $I(u)$ by the functional

$$
\begin{aligned}
I_{+}(u)= & \frac{1}{2} \int_{\mathbb{R}^{3}}\left|(-\Delta)^{\frac{s}{2}} u\right|^{2} d x+\frac{1}{2} \int_{\mathbb{R}^{3}} V(x) u^{2} d x+\frac{1}{2 q} \int_{\mathbb{R}^{3}} K(x) \phi_{u^{+}}^{t}\left|u^{+}\right|^{q} d x \\
& -\int_{\mathbb{R}^{3}} h(x) F\left(u^{+}\right) d x-\frac{1}{2_{s}^{\star}} \int_{\mathbb{R}^{3}}\left|u^{+}\right|^{2^{\star}} d x .
\end{aligned}
$$

By using $u_{0}^{-}$as a test function, we obtain

$$
0=\left\langle I_{+}^{\prime}\left(u_{0}\right), u_{0}^{-}\right\rangle=\left\|u_{0}^{-}\right\|^{2}+\int_{\mathbb{R}^{3}}(-\Delta)^{\frac{s}{2}} u_{0}^{+}(-\Delta)^{\frac{s}{2}} u_{0}^{-} d x
$$

Since $\int_{\mathbb{R}^{3}}(-\Delta)^{\frac{s}{2}} u_{0}^{+}(-\Delta)^{\frac{s}{2}} u_{0}^{-} d x \geq 0$, we have $\left\|u_{0}^{-}\right\|=0$ which implies that $u_{0} \geq 0$ in $\mathbb{R}^{3}$. Using the same argument as Proposition 5.1 in [35], we have $u_{0} \in L^{\infty}\left(\mathbb{R}^{3}\right)$. By standard argument to the proof of Proposition 4.4 in [32], using Proposition 2.9 in [27] twice, we have that $u_{0} \in C^{2, \alpha}\left(\mathbb{R}^{3}\right)$ for some $\alpha \in(0,1)$ for $s>\frac{1}{2}$. Furthermore, if $u_{0}(x)=0$ for some $x_{0} \in \mathbb{R}^{3}$, then $(-\Delta)^{s} u_{0}\left(x_{0}\right)=0$. Since $u_{0} \in C^{2, \alpha}\left(\mathbb{R}^{3}\right)$, by Lemma 3.2 [22] and $u_{0}\left(x_{0}\right)=0$, we have

$$
(-\Delta)^{s} u_{0}\left(x_{0}\right)=-\frac{C(s)}{2} \int_{\mathbb{R}^{3}} \frac{u_{0}\left(x_{0}+y\right)+u_{0}\left(x_{0}-y\right)}{|y|^{3+2 s}} d y=0 .
$$

It implies $u_{0} \equiv 0$, which is a contradiction. 


\section{Existence of sign-changing solutions}

In this section we study the existence of sign-changing solutions of problem (1.1) and prove Theorem 1.2. To begin with, we give some technical lemmas, which are crucial in the proof of Theorem 1.2.

Lemma 4.1. Under the assumptions of Theorem 1.2, then we have $0<m<c_{0}+\frac{s}{3} \mathcal{S}_{s}^{\frac{3}{2 s}}$.

Proof. By the definition of $\mathcal{M}$ and $\mathcal{N}$, we have $\mathcal{M} \subset \mathcal{N}$ and $m \geq c_{0}>0$. Now, we prove $m<c_{0}+\frac{s}{3} \mathcal{S}_{s}^{\frac{3}{2 s}}$. Since Lemma 2.7(iii) and (iv) hold, utilizing the same argument as [38], we know that there exist $a_{\varepsilon}, b_{\varepsilon}>0$ such that $a_{\varepsilon} u_{0}-b_{\varepsilon} u_{\varepsilon} \in \mathcal{M}$. Therefore, to finish the proof, it is enough to show that there is $\varepsilon>0$ small enough such that

$$
\sup _{a, b>0} I\left(a u_{0}-b u_{\varepsilon}\right)<c_{0}+\frac{s}{3} \mathcal{S}_{s}^{\frac{3}{2 s}}
$$

In fact, by the definition of $I\left(a u_{0}-b u_{\varepsilon}\right)$, it is easy to see that $I\left(a u_{0}-b u_{\varepsilon}\right)<0$ for $a$ or $b$ large enough. So it is sufficient to consider that $a \in\left(0, a^{\star}\right]$ and $b \in\left(0, b^{\star}\right]$ for some $a^{\star}, b^{\star}>0$. Moreover, by the continuity of $I$ with respect to $b$, there exists a constant $b_{0} \in\left(0, b^{\star}\right)$ such that

$$
I\left(a u_{0}-b u_{\varepsilon}\right)<I\left(a u_{0}\right)+\frac{s}{3} \delta_{s}^{\frac{3}{2 s}} \leq I\left(u_{0}\right)+\frac{s}{3} \delta_{s}^{\frac{3}{2 s}}=c_{0}+\frac{s}{3} \delta_{s}^{\frac{3}{2 s}},
$$

for any $a>0$ and $b \in\left(0, b_{0}\right)$. Then, we assume that $a \in\left(0, a^{\star}\right]$ and $b \in\left[b_{0}, b^{\star}\right]$. By a direct calculation, we have

$$
I\left(a u_{0}-b u_{\varepsilon}\right)=I\left(a u_{0}\right)+A_{1}+A_{2}+A_{3}+A_{4}+A_{5}+A_{6},
$$

where

$$
\begin{gathered}
A_{1}=\frac{1}{2} \int_{\mathbb{R}^{3}}\left|(-\Delta)^{\frac{s}{2}}\left(b u_{\varepsilon}\right)\right|^{2} d x-\frac{1}{2_{s}^{*}} \int_{\mathbb{R}^{3}}\left|b u_{\varepsilon}\right|^{2_{s}^{*}} d x, \\
A_{2}=\frac{1}{2_{s}^{\star}} \int_{\mathbb{R}^{3}}\left(\left|b u_{\varepsilon}\right|^{2_{s}^{*}}+\left|a u_{0}\right|^{2_{s}^{*}}-\left|a u_{0}-b u_{\varepsilon}\right|^{2_{s}^{*}}\right) d x, \\
A_{3}=\int_{\mathbb{R}^{3}} h(x)\left(F\left(a u_{0}\right)+F\left(b u_{\varepsilon}\right)-F\left(a u_{0}-b u_{\varepsilon}\right)\right) d x \\
A_{4}=\frac{1}{2} \int_{\mathbb{R}^{3}} V(x)\left|b u_{\varepsilon}\right|^{2} d x-\int_{\mathbb{R}^{3}} h(x) F\left(b u_{\varepsilon}\right) d x \\
A_{5}=-\int_{\mathbb{R}^{3}}(-\Delta)^{\frac{s}{2}}\left(a u_{0}\right)(-\Delta)^{\frac{s}{2}}\left(b u_{\varepsilon}\right) d x-\int_{\mathbb{R}^{3}} V(x)\left(a u_{0}\right)\left(b u_{\varepsilon}\right) d x,
\end{gathered}
$$

and

$$
A_{6}=\frac{1}{2 q} \int_{\mathbb{R}^{3}} K(x) \phi_{a u_{0}-b u_{\varepsilon}}^{t}\left|a u_{0}-b u_{\varepsilon}\right|^{q} d x-\frac{1}{2 q} \int_{\mathbb{R}^{3}} K(x) \phi_{a u_{0}}^{t}\left|a u_{0}\right|^{q} d x .
$$

By (3.10), we obtain

$$
A_{1} \leq \frac{s}{3} \mathcal{S}_{s}^{\frac{3}{2 s}}+O\left(\varepsilon^{3-2 s}\right)
$$

Moreover, from the Calculus Lemma [11]:

$$
|| \alpha-\left.\beta\right|^{q}-|\alpha|^{q}-|\beta|^{q} \mid \leq C\left(|\alpha|^{q-1}|\beta|+|\alpha||\beta|^{q-1}\right), \quad \text { for any } \alpha, \beta \in \mathbb{R} \text { and } q \geq 1,
$$

By using $u_{0} \in L^{\infty}\left(\mathbb{R}^{3}\right)$ and (3.8), we infer that

$$
\begin{aligned}
A_{2} & \leq C \int_{\mathbb{R}^{3}}\left(\left|u_{\varepsilon}\right|^{2_{s}^{*}-1}\left|u_{0}\right|+\left|u_{\varepsilon} \| u_{0}\right|^{2_{s}^{*}-1}\right) d x \\
& \leq C\left\|u_{0}\right\|_{\infty} \int_{\mathbb{R}^{3}}\left|u_{\varepsilon}\right|^{2_{s}^{*}-1} d x+C\left\|u_{0}\right\|_{\infty}^{2_{s}^{*}-1} \int_{\mathbb{R}^{3}}\left|u_{\varepsilon}\right| d x \\
& =C\left\|u_{0}\right\|_{\infty} \int_{\mathbb{R}^{3}}\left|u_{\varepsilon}\right|^{2_{s}^{*}-1} d x+C\left\|u_{0}\right\|_{\infty}^{2_{s}^{*}-1} \int_{|x| \leq r_{0}}\left|u_{\varepsilon}\right| d x \\
& \leq C \int_{\mathbb{R}^{3}}\left|u_{\varepsilon}\right|^{2_{s}^{*}-1} d x+C\left(\int_{|x| \leq r_{0}}\left|u_{\varepsilon}\right|^{2} d x\right)^{\frac{1}{2}} \\
& \leq O\left(\varepsilon^{\frac{3-2 s}{2}}\right)+O\left(\varepsilon^{\frac{3-2 s}{2}}\right)=O\left(\varepsilon^{\frac{3-2 s}{2}}\right) .
\end{aligned}
$$


Firstly, we consider that $\left(V h_{2}\right)$ holds. Then, by (3.8), $\left(f_{1}\right)$ and $\left(f_{4}\right)$, we get

$$
\begin{aligned}
A_{3}= & \int_{\mathbb{R}^{3}} h(x)\left(b u_{\varepsilon} F^{\prime}\left(\theta\left(a u_{0}-b u_{\varepsilon}\right)+(1-\theta) a u_{0}\right)+F\left(b u_{\varepsilon}\right)\right) d x \\
\leq & b^{\star}\|h\|_{\infty} \int_{\mathbb{R}^{3}}\left|u_{\varepsilon}\right|\left|f\left(\theta\left(a u_{0}-b u_{\varepsilon}\right)+(1-\theta) a u_{0}\right)\right| d x \\
& +\|h\|_{\infty} \int_{\mathbb{R}^{3}}\left(C_{1}\left|b^{\star} u_{\varepsilon}\right|^{2 q}+C_{2}\left|b^{\star} u_{\varepsilon}\right|^{v}\right) d x \\
\leq & C \int_{\mathbb{R}^{3}}\left|u_{\varepsilon}\right|\left[\left|\theta\left(a u_{0}-b u_{\varepsilon}\right)+(1-\theta) a u_{0}\right|^{2 q-1}+\left|\theta\left(a u_{0}-b u_{\varepsilon}\right)+(1-\theta) a u_{0}\right|^{v-1}\right] d x \\
& +C \int_{\mathbb{R}^{3}}\left|u_{\varepsilon}\right|^{2 q} d x+C \int_{\mathbb{R}^{3}}\left|u_{\varepsilon}\right|^{v} d x \\
\leq & C \int_{\mathbb{R}^{3}}\left|u_{\varepsilon}\right|\left(\left|b u_{\varepsilon}\right|^{2 q-1}+\left|a u_{0}\right|^{2 q-1}+\left|b u_{\varepsilon}\right|^{v-1}+\left|a u_{0}\right|^{v-1}\right) d x \\
& +C \int_{\mathbb{R}^{3}}\left|u_{\varepsilon}\right|^{2 q} d x+C \int_{\mathbb{R}^{3}}\left|u_{\varepsilon}\right|^{v} d x \\
\leq & C \int_{\mathbb{R}^{3}}\left|u_{\varepsilon}\right| d x+C \int_{\mathbb{R}^{3}}\left|u_{\varepsilon}\right|^{2 q} d x+C \int_{\mathbb{R}^{3}}\left|u_{\varepsilon}\right|^{v} d x \\
\leq & O\left(\varepsilon^{\frac{3-2 s}{2}}\right)+O\left(\varepsilon^{3-q(3-2 s)}\right)+O\left(\varepsilon^{3-\frac{v(3-2 s)}{2}}\right),
\end{aligned}
$$

where $\theta \in(0,1)$.

Now, if $\left(V h_{3}\right)$ holds, then by $\left(\widetilde{f}_{1}\right),\left(f_{4}\right)$ and (3.8), we get

$$
\begin{aligned}
A_{3}= & \int_{\mathbb{R}^{3}} h(x)\left(b u_{\varepsilon} F^{\prime}\left(\theta\left(a u_{0}-b u_{\varepsilon}\right)+(1-\theta) a u_{0}\right)+F\left(b u_{\varepsilon}\right)\right) d x \\
\leq & b^{\star}|h(x)|_{\infty} \int_{\mathbb{R}^{3}}\left|u_{\varepsilon}\right|\left|f\left(\theta\left(a u_{0}-b u_{\varepsilon}\right)+(1-\theta) a u_{0}\right)\right| d x \\
& +|h(x)|_{\infty} \int_{\mathbb{R}^{3}}\left(C_{1}\left|b^{\star} u_{\varepsilon}\right|^{p}+C_{2}\left|b^{\star} u_{\varepsilon}\right|^{v}\right) d x \\
\leq & C \int_{\mathbb{R}^{3}}\left|u_{\varepsilon}\right|\left[\left|\theta\left(a u_{0}-b u_{\varepsilon}\right)+(1-\theta) a u_{0}\right|^{p-1}+\left|\theta\left(a u_{0}-b u_{\varepsilon}\right)+(1-\theta) a u_{0}\right|^{v-1}\right] d x \\
& +C \int_{\mathbb{R}^{3}}\left|u_{\varepsilon}\right|^{p} d x+C \int_{\mathbb{R}^{3}}\left|u_{\varepsilon}\right|^{v} d x \\
\leq & C \int_{\mathbb{R}^{3}}\left|u_{\varepsilon}\right|\left(\left|b u_{\varepsilon}\right|^{p-1}+\left|a u_{0}\right|^{p-1}+\left|b u_{\varepsilon}\right|^{v-1}+\left|a u_{0}\right|^{v-1}\right) d x \\
& +C \int_{\mathbb{R}^{3}}\left|u_{\varepsilon}\right|^{p} d x+C \int_{\mathbb{R}^{3}}\left|u_{\varepsilon}\right|^{v} d x \\
\leq & C \int_{\mathbb{R}^{3}}\left|u_{\varepsilon}\right| d x+C \int_{\mathbb{R}^{3}}\left|u_{\varepsilon}\right|^{p} d x+C \int_{\mathbb{R}^{3}}\left|u_{\varepsilon}\right|^{v} d x \\
\leq & O\left(\varepsilon^{\frac{3-2 s}{2}}\right)+O\left(\varepsilon^{3-\frac{p(3-2 s)}{2}}\right)+C_{1} \varepsilon^{3-\frac{v(3-2 s)}{2}},
\end{aligned}
$$

where $\theta \in(0,1)$.

From (3.8) and assumption $\left(f_{4}\right)$, we can see that

$$
\begin{aligned}
A_{4} & \leq \frac{1}{2} \int_{\mathbb{R}^{3}} V(x)\left|b^{\star} u_{\varepsilon}\right|^{2} d x-\lambda \int_{\mathbb{R}^{3}} h(x)\left|b_{0} u_{\varepsilon}\right|^{\mu} d x \\
& \leq C \int_{\mathbb{R}^{3}}\left|u_{\varepsilon}\right|^{2} d x-\lambda h_{0} b_{0} \int_{\mathbb{R}^{3}}\left|u_{\varepsilon}\right|^{\mu} d x \\
& \leq O\left(\varepsilon^{3-2 s}\right)-\lambda C_{2} \varepsilon^{3-\frac{\mu(3-2 s)}{2}} .
\end{aligned}
$$

Since $u_{0}$ is a positive solution of problem (1.1), it holds

$$
A_{5}=a^{2 q} b \int_{\mathbb{R}^{3}} K(x) \phi_{u_{0}}^{t}\left|u_{0}\right|^{q-1} u_{\varepsilon} d x-b \int_{\mathbb{R}^{3}} h(x) f\left(a u_{0}\right) u_{\varepsilon} d x-a^{2_{s}^{*}} b \int_{\mathbb{R}^{3}}\left|u_{0}\right|^{2_{s}^{*}-1} u_{\varepsilon} d x
$$


Using the Hölder's inequality, we obtain

$$
\begin{aligned}
a^{2 q} b \int_{\mathbb{R}^{3}} K(x) \phi_{u_{0}}^{t}\left|u_{0}\right|^{q-1} u_{\varepsilon} d x & \leq C\left\|\phi_{u_{0}}^{t}\right\|_{2_{t}^{*}}\left\|\left(u_{0}\right)^{q-1} u_{\varepsilon}\right\|_{\frac{6}{3+2 t}} \\
& \leq C\left\|u_{0}\right\|_{\infty}^{q-1}\left(\int_{\mathbb{R}^{3}}\left|u_{\varepsilon}\right|^{\frac{6}{3+2 t}} d x\right)^{\frac{3+2 t}{6}} \\
& =O\left(\varepsilon^{\frac{3-2 s}{2}}\right) .
\end{aligned}
$$

Thus, combining (4.3), (4.7) with (4.8), we obtain

$$
A_{5} \leq O\left(\varepsilon^{\frac{3-2 s}{2}}\right) .
$$

From (4.2)-(4.9), and note that

$$
3-\frac{v}{2}(3-2 s)<\min \left\{\frac{3-2 s}{2}, 3-\frac{p}{2}(3-2 s), 3-\frac{\mu}{2}(3-2 s), 3-q(3-2 s)\right\} .
$$

Therefore, to complete the proof it suffices to take $\lambda=C_{1} C_{2}^{-1} \varepsilon^{-\theta}$ with some $\theta>\frac{(v-\mu)}{2}(3-2 s)$ and prove that

$$
\lim _{\varepsilon \rightarrow 0^{+}} \frac{A_{6}}{\varepsilon^{3-\frac{v(3-2)}{2}}}=0
$$

Using again the Calculus Lemma [11]:

$$
|| \alpha-\left.\beta\right|^{q}-|\alpha|^{q}-|\beta|^{q} \mid \leq C\left(|\alpha|^{q-1}|\beta|+|\beta|^{q-1}|\alpha|\right), \quad \forall \alpha, \beta \in \mathbb{R}, q \geq 1,
$$

and by a direct computation, we get

$$
\begin{aligned}
A_{6}= & \frac{1}{2 q} \iint_{\mathbb{R}^{6}} K(x) K(y) \frac{\left|a u_{0}(x)-b u_{\varepsilon}(x)\right|^{q}\left|a u_{0}(y)-b u_{\varepsilon}(y)\right|^{q}-\left|a u_{0}(x)\right|^{q}\left|a u_{0}(y)\right|^{q}}{|x-y|^{3-2 t}} d x d y \\
\leq & C \iint_{\mathbb{R}^{6}} \frac{\left|u_{0}(x)\right|^{q-1} u_{\varepsilon}(x)\left|u_{0}(y)\right|^{q-1} u_{\varepsilon}(y)}{|x-y|^{3-2 t}} d x d y+C \iint_{\mathbb{R}^{6}} \frac{\left|u_{0}(x)\right|^{q-1} u_{\varepsilon}(x) u_{0}(y)\left|u_{\varepsilon}(y)\right|^{q-1}}{|x-y|^{3-2 t}} d x d y \\
& +C \iint_{\mathbb{R}^{6}} \frac{\left|u_{0}(x)\right|^{q-1} u_{\varepsilon}(x)\left|u_{0}(y)\right|^{q}}{|x-y|^{3-2 t}} d x d y+C \iint_{\mathbb{R}^{6}} \frac{\left|u_{0}(x)\right|^{q-1} u_{\varepsilon}(x)\left|u_{\varepsilon}(y)\right|^{q}}{|x-y|^{3-2 t}} d x d y \\
& +C \iint_{\mathbb{R}^{6}} \frac{u_{0}(x)\left|u_{\varepsilon}(x)\right|^{q-1}\left|u_{0}(y)\right|^{q-1} u_{\varepsilon}(y)}{|x-y|^{3-2 t}} d x d y+C \iint_{\mathbb{R}^{6}} \frac{u_{0}(x)\left|u_{\varepsilon}(x)\right|^{q-1} u_{0}(y)\left|u_{\varepsilon}(y)\right|^{q-1}}{|x-y|^{3-2 t}} d x d y \\
& +C \iint_{\mathbb{R}^{6}} \frac{u_{0}(x)\left|u_{\varepsilon}(x)\right|^{q-1}\left|u_{0}(y)\right|^{q}}{|x-y|^{3-2 t}} d x d y+C \iint_{\mathbb{R}^{6}} \frac{u_{0}(x)\left|u_{\varepsilon}(x)\right|^{q-1}\left|u_{\varepsilon}(y)\right|^{q}}{|x-y|^{3-2 t}} d x d y \\
& +C \iint_{\mathbb{R}^{6}} \frac{\left|u_{0}(x)\right|^{q}\left|u_{0}(y)\right|^{q-1} u_{\varepsilon}(y)}{|x-y|^{3-2 t}} d x d y+C \iint_{\mathbb{R}^{6}} \frac{\left|u_{0}(x)\right|^{q} u_{0}(y)\left|u_{\varepsilon}(y)\right|^{q-1}}{|x-y|^{3-2 t}} d x d y \\
& +C \iint_{\mathbb{R}^{6}} \frac{\left|u_{0}(x)\right|^{q}\left|u_{\varepsilon}(y)\right|^{q}}{|x-y|^{3-2 t}} d x d y \\
& +C \iint_{\mathbb{R}^{6}} \frac{\left|u_{\varepsilon}(x)\right|^{q}\left|u_{0}(y)\right|^{q-1} u_{\varepsilon}(y)}{|x-y|^{3-2 t}} d x d y+C \iint_{\mathbb{R}^{6}} \frac{\left|u_{\varepsilon}(x)\right|^{q} u_{0}(y)\left|u_{\varepsilon}(y)\right|^{q-1}}{|x-y|^{3-2 t}} d x d y \\
& +C \iint_{\mathbb{R}^{6}} \frac{\left.\left|u_{\varepsilon}(x)\right|\right|^{q}\left|u_{0}(y)\right|^{q}}{|x-y|^{3-2 t}} d x d y+C \iint_{\mathbb{R}^{6}} \frac{\left|u_{\varepsilon}(x)\right|^{q}\left|u_{\varepsilon}(y)\right|^{q}}{|x-y|^{3-2 t}} d x d y .
\end{aligned}
$$

Using the Hardy-Littlewood-Sobolev inequality and the Hölder's inequality, setting $\theta=6 /(3+2 t), \vartheta=6 q /(3+$ $2 t$ ) we have the following estimates:

$$
\begin{gathered}
\iint_{\mathbb{R}^{6}} \frac{\left|u_{0}(x)\right|^{q-1} u_{\varepsilon}(x)\left|u_{0}(y)\right|^{q-1} u_{\varepsilon}(y)}{|x-y|^{3-2 t}} d x d y \leq C\left\|u_{0}^{q-1} u_{\varepsilon}\right\|_{\theta}^{2} \leq C\left\|u_{0}\right\|_{9}^{2(q-1)}\left\|u_{\varepsilon}\right\|_{9}^{2} \leq C\left\|u_{\varepsilon}\right\|_{\vartheta}^{2}, \\
\iint_{\mathbb{R}^{6}} \frac{\left|u_{0}(x)\right|^{q-1} u_{\varepsilon}(x) u_{0}(y)\left|u_{\varepsilon}(y)\right|^{q-1}}{|x-y|^{3-2 t}} d x d y \leq C\left\|u_{0}\right\|_{9}^{q-1}\left\|u_{\varepsilon}\right\|_{9}\left\|u_{0}\right\|\left\|_{\vartheta}\right\| u_{\varepsilon}\left\|_{9}^{q-1} \leq C\right\| u_{\varepsilon} \|_{9}^{q},
\end{gathered}
$$




$$
\begin{aligned}
& \iint_{\mathbb{R}^{6}} \frac{\left|u_{0}(x)\right|^{q-1} u_{\varepsilon}(x)\left|u_{0}(y)\right|^{q}}{|x-y|^{3-2 t}} d x d y \leq\left\|u_{0}^{q-1} u_{\varepsilon}\right\|_{\theta}\left\|u_{0}^{q}\right\|_{\theta} \leq C\left\|u_{\varepsilon}\right\|_{\vartheta} . \\
& \iint_{\mathbb{R}^{6}} \frac{\left|u_{0}(x)\right|^{q-1} u_{\varepsilon}(x)\left|u_{\varepsilon}(y)\right|^{q}}{|x-y|^{3-2 t}} d x d y \leq\left\|u_{0}^{q-1} u_{\varepsilon}\right\|_{\theta}\left\|u_{\varepsilon}^{q}\right\|_{\theta} \leq C\left\|u_{\varepsilon}\right\|_{\vartheta}^{q+1} . \\
& \iint_{\mathbb{R}^{6}} \frac{u_{0}(x)\left|u_{\varepsilon}(x)\right|^{q-1}\left|u_{0}(y)\right|^{q-1} u_{\varepsilon}(y)}{|x-y|^{3-2 t}} d x d y \leq C\left\|u_{0} u_{\varepsilon}^{q-1}\right\|_{\theta}\left\|u_{0}^{q-1} u_{\varepsilon}\right\|_{\theta} \leq C\left\|u_{\varepsilon}\right\|_{\vartheta}^{q} . \\
& \iint_{\mathbb{R}^{6}} \frac{u_{0}(x)\left|u_{\varepsilon}(x)\right|^{q-1} u_{0}(y)\left|u_{\varepsilon}(y)\right|^{q-1}}{|x-y|^{3-2 t}} d x d y \leq C\left\|u_{0} u_{\varepsilon}^{q-1}\right\|_{\theta}\left\|u_{0} u_{\varepsilon}^{q-1}\right\|_{\theta} \leq C\left\|u_{\varepsilon}\right\|_{\vartheta}^{2 q-2} \text {. } \\
& \iint_{\mathbb{R}^{6}} \frac{u_{0}(x)\left|u_{\varepsilon}(x)\right|^{q-1}\left|u_{0}(y)\right|^{q}}{|x-y|^{3-2 t}} d x d y \leq C\left\|u_{0} u_{\varepsilon}^{q-1}\right\|_{\theta}\left\|u_{0}^{q}\right\|_{\theta} \leq C\left\|u_{\varepsilon}\right\|_{\vartheta}^{q-1} . \\
& \iint_{\mathbb{R}^{6}} \frac{u_{0}(x)\left|u_{\varepsilon}(x)\right|^{q-1}\left|u_{\varepsilon}(y)\right|^{q}}{|x-y|^{3-2 t}} d x d y \leq C\left\|u_{0} u_{\varepsilon}^{q-1}\right\|_{\theta}\left\|u_{\varepsilon}^{q}\right\|_{\theta} \leq C\left\|u_{\varepsilon}\right\|_{\vartheta}^{2 q-1} . \\
& \iint_{\mathbb{R}^{6}} \frac{\left|u_{0}(x)\right|^{q}\left|u_{0}(y)\right|^{q-1} u_{\varepsilon}(y)}{|x-y|^{3-2 t}} d x d y \leq C\left\|u_{0}^{q-1} u_{\varepsilon}\right\|_{\theta}\left\|u_{0}^{q}\right\|_{\theta} \leq C\left\|u_{\varepsilon}\right\|_{\vartheta} . \\
& \iint_{\mathbb{R}^{6}} \frac{\left|u_{0}(x)\right|^{q} u_{0}(y)\left|u_{\varepsilon}(y)\right|^{q-1}}{|x-y|^{3-2 t}} d x d y \leq C\left\|u_{0} u_{\varepsilon}^{q-1}\right\|_{\theta}\left\|u_{0}^{q}\right\|_{\theta} \leq C\left\|u_{\varepsilon}\right\|_{\vartheta}^{q-1} \text {. } \\
& \iint_{\mathbb{R}^{6}} \frac{\left|u_{\varepsilon}(x)\right|^{q}\left|u_{0}(y)\right|^{q}}{|x-y|^{3-2 t}} d x d y=\iint_{\mathbb{R}^{6}} \frac{\left|u_{0}(x)\right|^{q}\left|u_{\varepsilon}(y)\right|^{q}}{|x-y|^{3-2 t}} d x d y \leq C\left\|u_{\varepsilon}^{q}\right\|_{\theta}\left\|u_{0}^{q}\right\|_{\theta} \leq C\left\|u_{\varepsilon}\right\|_{g}^{q} \text {. } \\
& \iint_{\mathbb{R}^{6}} \frac{\left|u_{\varepsilon}(x)\right|^{q}\left|u_{0}(y)\right|^{q-1} u_{\varepsilon}(y)}{|x-y|^{3-2 t}} d x d y \leq C\left\|u_{\varepsilon}^{q}\right\|_{\theta}\left\|u_{0}^{q-1} u_{\varepsilon}\right\|_{\theta} \leq C\left\|u_{\varepsilon}\right\|_{\vartheta}^{q+1} . \\
& \iint_{\mathbb{R}^{6}} \frac{\left|u_{\varepsilon}(x)\right|^{q} u_{0}(y)\left|u_{\varepsilon}(y)\right|^{q-1}}{|x-y|^{3-2 t}} d x d y \leq C\left\|u_{\varepsilon}^{q}\right\|_{\theta}\left\|u_{0} u_{\varepsilon}^{q-1}\right\|_{\theta} \leq C\left\|u_{\varepsilon}\right\|_{\vartheta}^{2 q-1} . \\
& \iint_{\mathbb{R}^{6}} \frac{\left|u_{\varepsilon}(x)\right|^{q}\left|u_{\varepsilon}(y)\right|^{q}}{|x-y|^{3-2 t}} d x d y \leq C\left\|u_{\varepsilon}^{q}\right\|_{\theta}^{2} \leq C\left\|u_{\varepsilon}\right\|_{\theta}^{2 q} .
\end{aligned}
$$

Since $v>\frac{9 q-3-2 s q-2 t}{q(3-2 s)}$ and $q>\max \left\{\frac{3+2 t}{2(3-2 s)}, 2\right\}$, we have that

$$
\lim _{\varepsilon \rightarrow 0^{+}} \frac{\left\|u_{\varepsilon}\right\|_{\vartheta}}{\varepsilon^{3-\frac{v(3-2 s)}{2}}}=\lim _{\varepsilon \rightarrow 0^{+}} \frac{\left(\int_{\mathbb{R}^{3}}\left|u_{\varepsilon}\right|^{\frac{6 q}{3+2 t}} d x\right)^{\frac{3+2 t}{6 q}}}{\varepsilon^{3-\frac{v(3-2 s)}{2}}}=\lim _{\varepsilon \rightarrow 0^{+}} \frac{O\left(\varepsilon^{\frac{2 s q+2 t+3-3 q}{2 q}}\right)}{\varepsilon^{3-\frac{v(3-2 s)}{2}}}=0 .
$$

and thus for any $\tau \in\{2, q-1, q, q+1,2 q-2,2 q-1,2 q, 2 q+1\}$, one has

$$
\lim _{\varepsilon \rightarrow 0^{+}} \frac{\left\|u_{\varepsilon}\right\|_{\vartheta}^{\tau}}{\varepsilon^{3-\frac{v(3-2 s)}{2}}}=0
$$

Therefore, it follows from (4.8)-(4.12) and (4.6) that

$$
\lim _{\varepsilon \rightarrow 0^{+}} \frac{A_{6}}{\varepsilon^{3-\frac{v(3-2 s)}{2}}}=0 .
$$

Hence, (4.1) holds and we complete the proof.

Let $G(u, v)$ be the functional defined in $H$ by

$$
G(u, v)= \begin{cases}\frac{\int_{\mathbb{R}^{3}} h(x) f(u) u d x+\int_{\mathbb{R}^{3}}|u|^{2^{*}} d x}{\|u\|^{2}+\int_{\mathbb{R}^{3}}(-\Delta)^{\frac{s}{2}} u(-\Delta)^{\frac{s}{2}} v d x+\int_{\mathbb{R}^{3}} K(x) \phi_{u}^{t}|u|^{q} d x+\int_{\mathbb{R}^{3}} K(x) \phi_{u}^{t}|v|^{q} d x}, & \text { if } u \neq 0, \\ 0, & \text { if } u=0 .\end{cases}
$$

Denote by

$$
Q=\left\{u \in H:\left|G\left(u^{+}, u^{-}\right)-1\right|<\frac{1}{2},\left|G\left(u^{-}, u^{+}\right)-1\right|<\frac{1}{2}\right\} .
$$

It follows from the definition of $\mathcal{M}$ and $H$ that $u \in \mathcal{M}$ if and only if $G\left(u^{+}, u^{-}\right)=G\left(u^{-}, u^{+}\right)=1$. 
Lemma 4.2. For any $c \in\left(0, c_{0}+\frac{s}{3} \mathcal{S}_{s}^{\frac{3}{2 s}}\right)$, and if $\left\{u_{n}\right\} \subset Q$ satisfies

$$
I\left(u_{n}\right) \rightarrow c, \quad I^{\prime}\left(u_{n}\right) \rightarrow 0,
$$

then $\left\{u_{n}\right\}$ contains a convergent subsequence.

Proof. In view of Lemma 3.1, we see that $\left\{u_{n}\right\}$ is bounded in $H$. Therefore, there exists a subsequence of $\left\{u_{n}\right\}$, still denoted by $\left\{u_{n}\right\}$ such that

$$
\left\{\begin{array}{l}
u_{n} \rightarrow u \text { in } H, \\
u_{n} \rightarrow u \text { in } L_{l o c}^{r}\left(\mathbb{R}^{3}\right), \text { for } 1 \leq r<2_{s}^{\star}, \\
u_{n}(x) \rightarrow u(x) \text { a.e. in } \mathbb{R}^{3} .
\end{array}\right.
$$

Let $v_{n}=u_{n}-u$, then $v_{n} \rightarrow 0$ in $H$. Then, in view of Propositions 2.2-2.4 and the Brezis-Lieb Lemma, it is easy to check that $I^{\prime}(u)=0$ and so,

$$
\begin{aligned}
C= & I\left(u_{n}\right)+o_{n}(1) \\
= & \frac{1}{2}\left\|u_{n}\right\|^{2}+\frac{1}{2 q} \int_{\mathbb{R}^{3}} K(x) \phi_{u_{n}}^{t}\left|u_{n}\right|^{q} d x-\int_{\mathbb{R}^{3}} h(x) F\left(u_{n}\right) d x-\frac{1}{2_{s}^{\star}} \int_{\mathbb{R}^{3}}\left|u_{n}\right|^{2_{s}^{\star}} d x+o_{n}(1) \\
= & \frac{1}{2}\left(\left\|v_{n}\right\|^{2}+\|u\|^{2}\right)+\frac{1}{2 q} \int_{\mathbb{R}^{3}} K(x) \phi_{u}^{t}|u|^{q} d x-\int_{\mathbb{R}^{3}} h(x) F(u) d x \\
& -\frac{1}{2_{s}^{\star}} \int_{\mathbb{R}^{3}}\left|v_{n}\right|^{2_{s}^{\star}} d x-\frac{1}{2_{s}^{\star}} \int_{\mathbb{R}^{3}}|u|^{2_{s}^{\star}} d x+o_{n}(1) \\
= & I(u)+\frac{1}{2}\left\|v_{n}\right\|^{2}-\frac{1}{2_{s}^{\star}} \int_{\mathbb{R}^{3}}\left|v_{n}\right|^{2_{s}^{\star}} d x+o_{n}(1) \\
= & I(u)+\left(\frac{1}{2}\left\|v_{n}^{+}\right\|^{2}-\frac{1}{2_{s}^{\star}} \int_{\mathbb{R}^{3}}\left|v_{n}^{+}\right|^{2_{s}^{\star}} d x\right)+\left(\frac{1}{2}\left\|v_{n}^{-}\right\|^{2}-\frac{1}{2_{s}^{\star}} \int_{\mathbb{R}^{3}}\left|v_{n}^{-}\right|^{2_{s}^{\star}} d x\right) \\
& +\int_{\mathbb{R}^{3}}(-\Delta)^{\frac{s}{2}} v_{n}^{+}(-\Delta)^{\frac{s}{2}} v_{n}^{-} d x+o_{n}(1) .
\end{aligned}
$$

We know that either $v_{n}^{ \pm}=u_{n}^{ \pm}-u^{ \pm}$or $v_{n}^{ \pm}=u_{n}^{\mp}-u^{\mp}$ for $n$ large enough. Without loss of generality, assume that $v_{n}^{ \pm}=u_{n}^{ \pm}-u^{ \pm}$, then from $v_{n}^{ \pm} \rightarrow 0$ in $H$ we see that

$$
\begin{aligned}
\int_{\mathbb{R}^{3}}(-\Delta)^{\frac{s}{2}} u_{n}^{+}(-\Delta)^{\frac{s}{2}} u_{n}^{-} d x= & \int_{\mathbb{R}^{3}}(-\Delta)^{\frac{s}{2}} v_{n}^{+}(-\Delta)^{\frac{s}{2}} v_{n}^{-} d x+\int_{\mathbb{R}^{3}}(-\Delta)^{\frac{s}{2}} v_{n}^{+}(-\Delta)^{\frac{s}{2}} u^{-} d x \\
& +\int_{\mathbb{R}^{3}}(-\Delta)^{\frac{s}{2}} u^{+}(-\Delta)^{\frac{s}{2}} v_{n}^{-} d x+\int_{\mathbb{R}^{3}}(-\Delta)^{\frac{s}{2}} u^{+}(-\Delta)^{\frac{s}{2}} u^{-} d x \\
= & \int_{\mathbb{R}^{3}}(-\Delta)^{\frac{s}{2}} v_{n}^{+}(-\Delta)^{\frac{s}{2}} v_{n}^{-} d x+\int_{\mathbb{R}^{3}}(-\Delta)^{\frac{s}{2}} u^{+}(-\Delta)^{\frac{s}{2}} u^{-} d x+o_{n}(1) .
\end{aligned}
$$

Therefore, we have

$$
\begin{aligned}
0= & \left\langle I^{\prime}\left(u_{n}\right), u_{n}^{+}\right\rangle+o_{n}(1) \\
= & \left\|u_{n}^{+}\right\|^{2}+\int_{\mathbb{R}^{3}}(-\Delta)^{\frac{s}{2}} u_{n}^{+}(-\Delta)^{\frac{s}{2}} u_{n}^{-} d x+\int_{\mathbb{R}^{3}} K(x) \phi_{u_{n}}^{t}\left|u_{n}^{+}\right|^{q} d x \\
& -\int_{\mathbb{R}^{3}} h(x) f\left(u_{n}^{+}\right) u_{n}^{+} d x-\int_{\mathbb{R}^{3}}\left|u_{n}^{+}\right|^{2^{*}} d x+o_{n}(1) \\
= & \left(\left\|v_{n}^{+}\right\|^{2}+\left\|u^{+}\right\|^{2}\right)+\left(\int_{\mathbb{R}^{3}}(-\Delta)^{\frac{s}{2}} v_{n}^{+}(-\Delta)^{\frac{s}{2}} v_{n}^{-} d x+\int_{\mathbb{R}^{3}}(-\Delta)^{\frac{s}{2}} u^{+}(-\Delta)^{\frac{s}{2}} u^{-} d x\right) \\
& +\int_{\mathbb{R}^{3}} K(x) \phi_{u}^{t}\left|u^{+}\right|^{q} d x-\int_{\mathbb{R}^{3}} h(x) f\left(u^{+}\right) u^{+} d x-\left(\int_{\mathbb{R}^{3}}\left|v_{n}^{+}\right|^{2_{s}^{*}} d x+\int_{\mathbb{R}^{3}}\left|u^{+}\right|^{2^{*}} d x\right)+o_{n}(1) \\
= & \left\langle I^{\prime}(u), u^{+}\right\rangle+\left\|v_{n}^{+}\right\|^{2}+\int_{\mathbb{R}^{3}}(-\Delta)^{\frac{s}{2}} v_{n}^{+}(-\Delta)^{\frac{s}{2}} v_{n}^{-} d x-\int_{\mathbb{R}^{3}}\left|v_{n}^{+}\right|^{2_{s}^{*}} d x+o_{n}(1) \\
= & \left\|v_{n}^{+}\right\|^{2}+\int_{\mathbb{R}^{3}}(-\Delta)^{\frac{s}{2}} v_{n}^{+}(-\Delta)^{\frac{s}{2}} v_{n}^{-} d x-\int_{\mathbb{R}^{3}}\left|v_{n}^{+}\right|^{2_{s}^{*}} d x+o_{n}(1) .
\end{aligned}
$$


Similarly, we can obtain

$$
0=\left\langle I^{\prime}\left(u_{n}\right), u_{n}^{-}\right\rangle=\left\|v_{n}^{-}\right\|^{2}+\int_{\mathbb{R}^{3}}(-\Delta)^{\frac{s}{2}} v_{n}^{+}(-\Delta)^{\frac{s}{2}} v_{n}^{-} d x-\int_{\mathbb{R}^{3}}\left|v_{n}^{-}\right|^{2_{s}^{*}} d x+o_{n}(1) .
$$

If $v_{n} \rightarrow 0$ in $H$, the conclusion follows. So by contradiction, we assume that $v_{n} \rightarrow 0$ in $H$. Now, we consider three cases, respectively.

Case 1. $v_{n}^{+} \rightarrow 0\left(v_{n}^{+} \nrightarrow 0\right)$ in $H, v_{n}^{-} \rightarrow 0$ in $H$. In this case, $v_{n}^{-} \rightarrow 0$ in $H$ implies that $u_{n}^{-} \rightarrow u^{-}$in $H$. Since $\left\{u_{n}\right\} \subset Q$, we see that $u_{n}^{-} \neq 0$ and $\frac{3}{2}>H\left(u_{n}^{-}, u_{n}^{+}\right)>\frac{1}{2}$, thus,

$$
\begin{aligned}
\frac{1}{2}\left\|u_{n}^{-}\right\|^{2} & \leq \int_{\mathbb{R}^{3}} h(x) f\left(u_{n}^{-}\right) u_{n}^{-} d x+\int_{\mathbb{R}^{3}}\left|u_{n}^{-}\right|^{2_{s}^{*}} d x \\
& \leq C\left\|u_{n}^{-}\right\|^{2 q}+C\left\|u_{n}^{-}\right\|^{p}+C\left\|u_{n}^{-}\right\|^{v}+C\left\|u_{n}^{-}\right\|^{2_{s}^{*}},
\end{aligned}
$$

which implies that there exists some constant $C_{0}>0$ such that $\left\|u_{n}^{-}\right\| \geq C_{0}$, and

$$
\left\|u^{-}\right\|=\lim _{n \rightarrow \infty}\left\|u_{n}^{-}\right\| \geq C_{0}>0,
$$

and so $u \neq 0$. In view of $I^{\prime}(u)=0$ and $u \neq 0$, we get that $u \in \mathcal{N}$ and $I(u) \geq c_{0}$.

From $v_{n}^{+} \rightarrow 0\left(v_{n}^{+} \nrightarrow 0\right)$ in $H$, by (4.16), we can assume that for $n$ large enough

$$
\left\|v_{n}^{+}\right\|^{2}+\int_{\mathbb{R}^{3}}(-\Delta)^{\frac{s}{2}} v_{n}^{+}(-\Delta)^{\frac{s}{2}} v_{n}^{-} d x \rightarrow \ell>0, \int_{\mathbb{R}^{3}}\left|v_{n}^{+}\right|^{2_{s}^{*}} d x \rightarrow \ell>0 .
$$

By Sobolev inequality, we deduce that

$$
\int_{\mathbb{R}^{3}}\left|v_{n}^{+}\right|^{2_{s}^{*}} d x \leq \mathcal{S}_{s}^{-\frac{2_{s}^{*}}{2}}\left\|v_{n}^{+}\right\|^{2_{s}^{*}} \leq \mathcal{S}_{s}^{-\frac{2_{s}^{*}}{2}}\left(\left\|v_{n}^{+}\right\|^{2}+\int_{\mathbb{R}^{3}}(-\Delta)^{\frac{s}{2}} V_{n}^{+}(-\Delta)^{\frac{s}{2}} V_{n}^{-} d x\right)^{\frac{2_{s}^{*}}{2}},
$$

which implies that $\ell \geq \delta_{s}^{\frac{3}{2 s}}$. Therefore, we have

$$
\frac{1}{2}\left(\left\|v_{n}^{+}\right\|^{2}+\int_{\mathbb{R}^{3}}(-\Delta)^{\frac{s}{2}} v_{n}^{+}(-\Delta)^{\frac{s}{2}} V_{n}^{-} d x\right)-\frac{1}{2_{s}^{\star}} \int_{\mathbb{R}^{3}}\left|v_{n}^{+}\right|^{2_{s}^{\star}} d x=\frac{s}{3} \ell \geq \frac{s}{3} \delta_{s}^{\frac{3}{2 s}}
$$

Then, it follows from (4.15) and $v_{n}^{-} \rightarrow 0$ in $H$ that

$$
\begin{aligned}
c= & I\left(u_{n}\right)+o_{n}(1)=I(u)+\frac{1}{2}\left(\left\|v_{n}^{+}\right\|^{2}+\int_{\mathbb{R}^{3}}(-\Delta)^{\frac{s}{2}} V_{n}^{+}(-\Delta)^{\frac{s}{2}} V_{n}^{-} d x\right) \\
& -\frac{1}{2_{s}^{\star}} \int_{\mathbb{R}^{3}}\left|v_{n}^{+}\right|^{2_{s}^{\star}} d x+o_{n}(1) \geq c_{0}+\frac{s}{3} \mathcal{S}_{s}^{\frac{3}{2 s}},
\end{aligned}
$$

which contradicts the assumption $c \in\left(0, c_{0}+\frac{s}{3} S_{s}^{\frac{3}{2 s}}\right)$.

Case 2. $v_{n}^{-} \rightarrow 0\left(v_{n}^{-} \nrightarrow 0\right)$ in $H, v_{n}^{+} \rightarrow 0$ in $H$. Analogous to the proof in Case 1 , so we omit it.

Case 3. $v_{n}^{ \pm} \rightarrow 0\left(v_{n}^{ \pm} \nrightarrow 0\right)$ in $H$. Arguing as the proof in Case 1, by (4.15) - (4.17), we obtain that

$$
\frac{1}{2}\left(\left\|v_{n}^{+}\right\|^{2}+\int_{\mathbb{R}^{3}}(-\Delta)^{\frac{s}{2}} v_{n}^{+}(-\Delta)^{\frac{s}{2}} v_{n}^{-} d x\right)-\frac{1}{2_{s}^{\star}} \int_{\mathbb{R}^{3}}\left|v_{n}^{+}\right|^{2_{s}^{*}} d x \geq \frac{s}{3} \delta_{s}^{\frac{3}{2 s}},
$$

and

$$
\frac{1}{2}\left(\left\|v_{n}^{-}\right\|^{2}+\int_{\mathbb{R}^{3}}(-\Delta)^{\frac{s}{2}} v_{n}^{+}(-\Delta)^{\frac{s}{2}} v_{n}^{-} d x\right)-\frac{1}{2_{s}^{\star}} \int_{\mathbb{R}^{3}}\left|v_{n}^{-}\right|^{2_{s}^{*}} d x \geq \frac{s}{3} \delta_{s}^{\frac{3}{2 s}} .
$$

It follows from $I^{\prime}(u)=0$ and $\left(f_{3}\right)$ that

$$
\begin{aligned}
I(u) & =I(u)-\frac{1}{2 q}\left\langle I^{\prime}(u), u\right\rangle \\
& =\frac{q-1}{2 q}\|u\|^{2}+\frac{1}{2 q} \int_{\mathbb{R}^{3}} h(x)(u f(u)-2 q F(u)) d x+\left(\frac{1}{2 q}-\frac{1}{2_{s}^{\star}}\right) \int_{\mathbb{R}^{3}}|u|^{2_{s}^{\star}} d x \\
& \geq \frac{q-1}{2 q}\|u\|^{2} \geq 0 .
\end{aligned}
$$


Therefore, by Lemma 3.2, Proposition 2.4 and (4.15), we infer that

$$
\begin{aligned}
c= & I\left(u_{n}\right)+o_{n}(1) \\
= & I(u)+\left(\frac{1}{2}\left\|v_{n}^{+}\right\|^{2}-\frac{1}{2_{s}^{\star}} \int_{\mathbb{R}^{3}}\left|v_{n}^{+}\right|^{2_{s}^{*}} d x\right)+\left(\frac{1}{2}\left\|v_{n}^{-}\right\|^{2}-\frac{1}{2_{s}^{\star}} \int_{\mathbb{R}^{3}}\left|v_{n}^{-}\right|^{2_{s}^{*}} d x\right) \\
& +\int_{\mathbb{R}^{3}}(-\Delta)^{\frac{s}{2}} v_{n}^{+}(-\Delta)^{\frac{s}{2}} v_{n}^{-} d x+o_{n}(1) \\
& \geq \frac{s}{3} \mathcal{S}_{s}^{\frac{3}{2 s}}+\frac{s}{3} \mathcal{S}_{s}^{\frac{3}{2 s}} \\
& >c_{0}+\frac{s}{3} \mathcal{S}_{s}^{\frac{3}{2 s}} .
\end{aligned}
$$

which contradicts the assumption $c \in\left(0, c_{0}+\frac{s}{3} \mathcal{S}_{s}^{\frac{3}{2 s}}\right)$.

Now let $P$ denote the cone of non-negative functions in $H, Q=[0,1] \times[0,1]$ and $\Sigma$ be the set of continuous maps $\sigma$ such that for each $\alpha, \beta \in[0,1]$

$$
\begin{aligned}
& \text { (a) } \sigma(\alpha, 0)=0, \sigma(0, \beta) \in P \text { and } \sigma(1, \beta) \in-P ; \\
& \text { (b) }(I \circ \sigma)(\alpha, 1) \leq 0, \frac{\int_{\mathbb{R}^{3}} h(x) f(\sigma(\alpha, 1)) \sigma(\alpha, 1) d x+\int_{\mathbb{R}^{3}}|\sigma(\alpha, 1)|^{2_{s}^{*}} d x}{\|\sigma(\alpha, 1)\|^{2}+\int_{\mathbb{R}^{3}} K(x) \phi_{\sigma(\alpha, 1)}^{t}|\sigma(\alpha, 1)|^{q} d x} \geq 2 .
\end{aligned}
$$

It is easy to check that for any $u \in H$ with $u^{ \pm} \neq 0, \sigma(\alpha, \beta)=\gamma \beta(1-\alpha) u^{+}+\gamma \alpha \beta u^{-} \in \Sigma$ for $\gamma>0$ large enough, which implies that $\Sigma \neq \emptyset$.

Lemma 4.3. $\inf _{\sigma \in \Sigma} \sup _{u \in \sigma(Q)} I(u)=\inf _{u \in \mathcal{M}} I(u)=m$.

Proof. Firstly, we claim that for any $\sigma \in \Sigma$, there exists $u_{1} \in \sigma(Q) \cap \mathcal{M}$ which implies that

$$
\sup _{u \in \sigma(Q)} I(u) \geq I\left(u_{1}\right) \geq \inf _{u \in \mathcal{M}} I(u),
$$

and thus

$$
\inf _{\sigma \in \Sigma} \sup _{u \in \sigma(Q)} I(u) \geq \inf _{u \in \mathcal{M}} I(u)
$$

In fact, for any $\sigma \in \Sigma$ and $\beta \in[0,1]$, it is easy to see that $\sigma(0, \beta) \in P$ and $\sigma(1, \beta) \in-P$. Hence, we have

$$
\begin{gathered}
G\left(\sigma^{+}(0, \beta), \sigma^{-}(0, \beta)\right)-G\left(\sigma^{-}(0, \beta), \sigma^{+}(0, \beta)\right)=G\left(\sigma^{+}(0, \beta), \sigma^{-}(0, \beta)\right) \geq 0 . \\
G\left(\sigma^{+}(1, \beta), \sigma^{-}(1, \beta)\right)-G\left(\sigma^{-}(1, \beta), \sigma^{+}(1, \beta)\right)=-G\left(\sigma^{-}(1, \beta), \sigma^{+}(1, \beta)\right) \leq 0 .
\end{gathered}
$$

Moreover, for all $\sigma \in \Sigma$ and $\alpha \in[0,1]$, by the elementary inequality $\frac{b}{a}+\frac{d}{c} \geq \frac{b+d}{a+c}$ for all $a, b, c, d>0$, the definition of $\Sigma$ and $\left(f_{3}\right)$, we get

$$
\begin{aligned}
& G\left(\sigma^{+}(\alpha, 1), \sigma^{-}(\alpha, 1)\right)+G\left(\sigma^{-}(\alpha, 1), \sigma^{+}(\alpha, 1)\right) \\
& \geq \frac{\int_{\mathbb{R}^{3}} h(x) f(\sigma(\alpha, 1)) \sigma(\alpha, 1) d x+\int_{\mathbb{R}^{3}}|\sigma(\alpha, 1)|^{2_{s}^{*}} d x}{\|\sigma(\alpha, 1)\|^{2}+\int_{\mathbb{R}^{3}} K(x) \phi_{\sigma(\alpha, 1)}^{t}|\sigma(\alpha, 1)|^{q} d x} \geq 2 .
\end{aligned}
$$

Therefore

$$
\begin{gathered}
G\left(\sigma^{+}(\alpha, 1), \sigma^{-}(\alpha, 1)\right)+G\left(\sigma^{-}(\alpha, 1), \sigma^{+}(\alpha, 1)\right)-2 \geq 0, \\
G\left(\sigma^{+}(\alpha, 0), \sigma^{-}(\alpha, 0)\right)+G\left(\sigma^{-}(\alpha, 0), \sigma^{+}(\alpha, 0)-2=-2 \leq 0 .\right.
\end{gathered}
$$

Therefore, by (4.20)-(4.23) and Miranda's Theorem, there exists $\left(\alpha^{\prime}, \beta^{\prime}\right) \in Q$ such that

$$
\begin{aligned}
0 & =G\left(\sigma^{+}\left(\alpha^{\prime}, \beta^{\prime}\right), \sigma^{-}\left(\alpha^{\prime}, \beta^{\prime}\right)\right)-G\left(\sigma^{-}\left(\alpha^{\prime}, \beta^{\prime}\right), \sigma^{+}\left(\alpha^{\prime}, \beta^{\prime}\right)\right) \\
& =G\left(\sigma^{+}\left(\alpha^{\prime}, \beta^{\prime}\right), \sigma^{-}\left(\alpha^{\prime}, \beta^{\prime}\right)\right)+G\left(\sigma^{-}\left(\alpha^{\prime}, \beta^{\prime}\right), \sigma^{+}\left(\alpha^{\prime}, \beta^{\prime}\right)-2 .\right.
\end{aligned}
$$


Thus

$$
G\left(\sigma^{+}\left(\alpha^{\prime}, \beta^{\prime}\right), \sigma^{-}\left(\alpha^{\prime}, \beta^{\prime}\right)\right)=G\left(\sigma^{-}\left(\alpha^{\prime}, \beta^{\prime}\right), \sigma^{+}\left(\alpha^{\prime}, \beta^{\prime}\right)\right)=1,
$$

which implies that for any $\sigma \in \Sigma$, there exists $u_{1}=\sigma\left(\alpha^{\prime}, \beta^{\prime}\right) \in \sigma(Q) \cap \mathcal{M}$.

On the other hand, for any $u \in \mathcal{M}$, there exists $\sigma(\alpha, \beta)=\gamma \beta(1-\alpha) u^{+}+\gamma \alpha \beta u^{-} \in \Sigma$ for $\gamma>0$ large enough. Therefore, by Propositions 2.2,2.4, we get

$$
I(u)=\max _{\alpha, \beta \geq 0} I\left(\alpha u^{+}+\beta u^{-}\right) \geq \sup _{u \in \sigma(Q)} I(u) \geq \inf _{\sigma \in \Sigma} \sup _{u \in \sigma(Q)} I(u),
$$

which implies that

$$
\inf _{u \in \mathcal{M}} I(u) \geq \inf _{\sigma \in \Sigma} \sup _{u \in \sigma(Q)} I(u) .
$$

Combining (4.19) with (4.24), we complete the proof.

Lemma 4.4. There exists $a(P S)_{d}$ sequence $\left\{u_{n}\right\} \subset Q$ for $I$.

Proof. Let $\left\{\widetilde{u}_{n}\right\} \subset \mathcal{M}$ be a minimizing sequence and denote by $\widetilde{\sigma}_{n}(\alpha, \beta)=\gamma \beta(1-\alpha) \widetilde{u}_{n}^{+}+\gamma \alpha \beta \widetilde{u}_{n}^{-} \in \Sigma$. Then, we have

$$
\lim _{n \rightarrow \infty} \max _{\widetilde{\sigma}_{n}(Q)} I(u)=\lim _{n \rightarrow \infty} I\left(\widetilde{u}_{n}\right)=d .
$$

Applying a result due to Lemma 1 [13], of the well-known deformation Lemma [24], we have that there exists $\left\{u_{n}\right\} \subset H$ such that

$$
I\left(u_{n}\right) \rightarrow d, I^{\prime}\left(u_{n}\right) \rightarrow 0, \operatorname{dist}\left(u_{n}, \widetilde{\sigma}_{n}(Q)\right) \rightarrow 0, \text { as } n \rightarrow \infty .
$$

Suppose by contradiction that the claim is not true, then it is possible to find a $\delta>0$ such that $\widetilde{\sigma}_{n}(Q) \cap U_{\delta}=\emptyset$ for $n$ large enough, where

$$
U_{\delta}=\left\{u \in H: \exists v \in H \text { such that }\|v-u\| \leq \delta,\left\|I^{\prime}(v)\right\| \leq \delta,|I(v)-d| \leq \delta\right\} .
$$

By [13], there exists a continuous map $\eta:[0,1] \times H \rightarrow H$ satisfying for some $\varepsilon \in\left(0, \frac{d}{2}\right)$ and all $\alpha \in[0,1]$

(i) $\eta(0, u)=u, \eta(\alpha,-u)=-\eta(\alpha, u)$;

(ii) $\eta(\alpha, u)=u, \forall u \in I^{d-\frac{\varepsilon}{2}} \cup\left(H \backslash I^{d+\frac{\varepsilon}{2}}\right)$;

(iii) $\eta\left(1, I^{d+\frac{\varepsilon}{2}} \backslash U_{\delta}\right) \subset I^{d-\frac{\varepsilon}{2}}$;

(iv) $\eta\left(1,\left(I^{d+\frac{\varepsilon}{2}} \cap P\right) \backslash U_{\delta}\right) \subset I^{d-\frac{\varepsilon}{2}} \cap P$; where $I^{c}=\{u \in H: I(u) \leq c\}$.

Since $\lim _{n \rightarrow \infty} \max _{\widetilde{\sigma}_{n}(Q)} I(u)=d$, we can take $n$ such that

$$
\widetilde{\sigma}_{n}(Q) \subset I^{d+\frac{\varepsilon}{2}}, \widetilde{\sigma}_{n}(Q) \cap U_{\delta}=\emptyset .
$$

Define

$$
\widehat{\sigma}_{n}: Q \rightarrow H
$$

by

$$
\widehat{\sigma}_{n}(\alpha, \beta)=\eta\left(1, \widetilde{\sigma}_{n}(\alpha, \beta)\right), \forall(\alpha, \beta) \in Q .
$$

Next we claim that $\widehat{\sigma}_{n} \in \Sigma$ and thus $\widehat{\sigma}_{n}(Q) \subset I^{d-\frac{\varepsilon}{2}}$, in view of (4.26) and the property (iii) of $\eta$, which yields a contradiction since

$$
d=\inf _{\sigma \in \Sigma} \sup _{u \in \sigma(Q)} I(u) \leq \max _{u \in \widehat{\sigma}_{n}(Q)} I(u) \leq d-\frac{\varepsilon}{2} .
$$

In fact, by the property (ii) of $\eta$ and $\widetilde{\sigma}_{n} \in \Sigma$, we have

$$
\widehat{\sigma}_{n}(\alpha, 0)=\eta\left(1, \widetilde{\sigma}_{n}(\alpha, 0)\right)=\eta(1,0)=0 .
$$

From (4.26), $\widehat{\sigma}_{n}(0, \beta) \in P$ and the property (iv) of $\eta$, we know that $\widehat{\sigma}_{n}(0, \beta) \in P$. By $\widetilde{\sigma}_{n}(1, \beta) \in-P$ and (4.26), we get

$$
-\widetilde{\sigma}_{n}(1, \beta) \in\left(I^{d+\frac{\varepsilon}{2}} \cap P\right) \backslash U_{\delta},
$$


which implies that $\widehat{\sigma}_{n}(1, \beta)=-\eta\left(1,-\widetilde{\sigma}_{n}(1, \beta)\right) \in-P$ by virtue of the properties (i) and (iv) of $\eta$. Thus, $\widehat{\sigma}_{n}$ satisfies the property $(a)$. On the other hand, $\left(I \circ \widetilde{\sigma}_{n}\right)(\alpha, 1) \leq 0$ and the property (ii) of $\eta$ imply that $\widehat{\sigma}_{n}(\alpha, 1)=$ $\eta\left(1, \widetilde{\sigma}_{n}(\alpha, 1)\right)=\widetilde{\sigma}_{n}(\alpha, 1)$. Thus, $\widehat{\sigma}_{n}$ satisfies the property $(b)$. Since $\eta$ and $\widetilde{\sigma}_{n}$ are continuous maps that $\widehat{\sigma}_{n}$ is continuous map, we see that $\widehat{\sigma}_{n} \in \Sigma$.

Finally, we show that $\left\{u_{n}\right\} \subset Q$ for $n$ large enough. Since $I^{\prime}\left(u_{n}\right) \rightarrow 0$, we have $\left\langle I^{\prime}\left(u_{n}\right), u_{n}^{ \pm}\right\rangle=o_{n}(1)$. Hence we only need to prove that $u_{n}^{ \pm} \neq 0$, which implies that $G\left(u_{n}^{+}, u_{n}^{-}\right) \rightarrow 1$ and $G\left(u_{n}^{-}, u_{n}^{+}\right) \rightarrow 1$, and so $\left\{u_{n}\right\} \subset Q$ for $n$ large enough. By (4.25), there exists a sequence $\left\{v_{n}\right\}$ such that

$$
v_{n}=\alpha_{n} \widetilde{u}_{n}^{+}+\beta_{n} \widetilde{u}_{n}^{-} \in \widetilde{\sigma}_{n}(Q), \quad\left\|v_{n}-u_{n}\right\| \rightarrow 0 .
$$

Hence, in order to get $u_{n}^{ \pm} \neq 0$, we can show that $\alpha_{n} \widetilde{u}_{n}^{+} \neq 0$ and $\beta_{n} \widetilde{u}_{n}^{-} \neq 0$ for $n$ large enough. By $\left\{\widetilde{u}_{n}\right\} \subset \mathcal{M}$, either $\left(f_{1}\right)$ or $\left(\widetilde{f}_{1}\right),\left(f_{2}\right)$ and the Sobolev embedding inequality, we deduce that

$$
\begin{aligned}
\left\|\widetilde{u}_{n}^{+}\right\|^{2} & \leq\left\|\widetilde{u}_{n}^{+}\right\|^{2}+\int_{\mathbb{R}^{3}}(-\Delta)^{\frac{s}{2}} \widetilde{u}_{n}^{+}(-\Delta)^{\frac{s}{2}} \widetilde{u}_{n}^{-} d x+\int_{\mathbb{R}^{3}} K(x) \phi_{\widetilde{u}_{n}}^{t}\left|\widetilde{u}_{n}^{+}\right|^{q} d x \\
& =\int_{\mathbb{R}^{3}} h(x) f\left(\widetilde{u}_{n}^{+}\right) u_{n}^{+} d x+\int_{\mathbb{R}^{3}}\left|\widetilde{u}_{n}^{+}\right|^{2_{s}^{*}} d x \\
& \leq C\left\|\widetilde{u}_{n}^{+}\right\|^{2 q}+C\left\|\widetilde{u}_{n}^{+}\right\|^{p}+C\left\|\widetilde{u}_{n}^{+}\right\|^{v}+C\left\|\widetilde{u}_{n}^{+}\right\|^{2_{s}^{*}},
\end{aligned}
$$

which implies that $\left\|\widetilde{u}_{n}^{+}\right\| \geq C_{1}$ for some constant $C_{1}>0$. It follows from $\left\{\widetilde{u}_{n}\right\} \subset \mathcal{M} \subset \mathcal{N}$ and $\left(f_{3}\right)$ that

$$
\begin{aligned}
d+o_{n}(1) & \geq I\left(\widetilde{u}_{n}\right)-\frac{1}{2 q}\left\langle I^{\prime}\left(\widetilde{u}_{n}\right), \widetilde{u}_{n}\right\rangle \\
& =\frac{q-1}{2 q}\left\|\widetilde{u}_{n}\right\|^{2}+\frac{1}{2 q} \int_{\mathbb{R}^{3}} h(x)\left(\widetilde{u}_{n} f\left(\widetilde{u}_{n}\right)-2 q F\left(\widetilde{u}_{n}\right)\right) d x+\left(\frac{1}{2 q}-\frac{1}{2_{s}^{\star}}\right) \int_{\mathbb{R}^{3}}\left|\widetilde{u}_{n}\right|^{2_{s}^{\star}} d x \\
& \geq \frac{q-1}{2 q}\left\|\widetilde{u}_{n}\right\|^{2} \geq \frac{q-1}{2 q}\left\|\widetilde{u}_{n}^{+}\right\|^{2},
\end{aligned}
$$

which implies that $d>0$ and $\left\|\widetilde{u}_{n}^{+}\right\| \leq C_{2}$ for some constant $C_{2}>0$. Thus, $C_{1} \leq\left\|\widetilde{u}_{n}^{+}\right\| \leq C_{2}$. Similarly, we can show that $C_{3} \leq\left\|u_{n}^{-}\right\| \leq C_{4}$ for some constant $C_{3}, C_{4}>0$. Therefore, we only need to prove that $\alpha_{n} \nrightarrow 0$ and $\beta_{n} \nrightarrow 0$. In fact, suppose that $\alpha_{n} \rightarrow 0$, it follows from the continuity of $I$ and (4.27) that

$$
d=\lim _{n \rightarrow \infty} I\left(v_{n}\right)=\lim _{n \rightarrow \infty} I\left(\alpha_{n} \widetilde{u}_{n}^{+}+\beta_{n} \widetilde{u}_{n}^{-}\right)=\lim _{n \rightarrow \infty} I\left(\beta_{n} \widetilde{u}_{n}^{-}\right) .
$$

But for $\alpha$ small enough, we have

$$
\begin{aligned}
d= & \lim _{n \rightarrow \infty} I\left(\widetilde{u}_{n}\right)=\lim _{n \rightarrow \infty} \max _{\alpha, \beta>0} I\left(\alpha \widetilde{u}_{n}^{+}+\beta \widetilde{u}_{n}^{-}\right) \geq \lim _{n \rightarrow \infty} \max _{\alpha>0} I\left(\alpha \widetilde{u}_{n}^{+}+\beta_{n} \widetilde{u}_{n}^{-}\right) \\
= & \lim _{n \rightarrow \infty} \max _{\alpha>0}\left[\frac{\alpha^{2}}{2}\left\|\widetilde{u}_{n}^{+}\right\|^{2}+\alpha \beta_{n} \int_{\mathbb{R}^{3}}(-\Delta)^{\frac{s}{2}} \widetilde{u}_{n}^{+}(-\Delta)^{\frac{s}{2}} \widetilde{u}_{n}^{-} d x\right. \\
& \left.\quad-\int_{\mathbb{R}^{3}} h(x) F\left(\alpha \widetilde{u}_{n}^{+}\right) d x-\frac{\alpha^{2_{s}^{*}}}{2_{s}^{\star}} \int_{\mathbb{R}^{3}}\left|\widetilde{u}_{n}^{+}\right|^{2_{s}^{*}} d x\right]+\lim _{n \rightarrow \infty} I\left(\beta_{n} \widetilde{u}_{n}^{-}\right) \\
\geq & \lim _{n \rightarrow \infty} \max _{\alpha>0}\left[\frac{\alpha^{2}}{2}\left\|\widetilde{u}_{n}^{+}\right\|^{2}-C\left(\alpha^{2 q}\left\|\widetilde{u}_{n}^{+}\right\|^{2 q}+\alpha^{p}\left\|\widetilde{u}_{n}^{+}\right\|^{p}+\alpha^{v}\left\|\widetilde{u}_{n}^{+}\right\|^{v}+\alpha^{2_{s}^{*}}\left\|\widetilde{u}_{n}^{+}\right\|^{2_{s}^{*}}\right)\right]+d \\
\geq & \max _{\alpha>0}\left[C_{1} \alpha^{2}-C_{2}\left(\alpha^{2 q}+\alpha^{p}+\alpha^{v}+\alpha^{2_{s}^{*}}\right)\right]+d \\
> & d,
\end{aligned}
$$

which is a contradiction. Thus, $\left\{u_{n}\right\} \subset Q$ for $n$ large enough.

Proof of Theorem 1.2. By Lemma 4.4, there exists a sequence $\left\{u_{n}\right\} \subset Q$ such that $I\left(u_{n}\right) \rightarrow d, I^{\prime}\left(u_{n}\right) \rightarrow 0$. By Lemma 4.1 and Lemma 4.2, we deduce that $\left\{u_{n}\right\}$ contains a convergent subsequence, still denoted by $\left\{u_{n}\right\}$. We assume that $u_{n} \rightarrow u$ in $H$ as $n \rightarrow \infty$ and thus $I(u)=d, I^{\prime}(u)=0$. Moreover, from $\left\{u_{n}\right\} \subset Q$ and the definition of $Q$, we have that $\frac{1}{2}<G\left(u_{n}^{+}, u_{n}^{-}\right)<\frac{3}{2}, \frac{1}{2}<G\left(u_{n}^{-}, u_{n}^{+}\right)<\frac{3}{2}$. Similar to the proof of (4.18), we infer that

$$
\left\|u^{ \pm}\right\|=\lim _{n \rightarrow \infty}\left\|u_{n}^{ \pm}\right\| \geq C_{1}>0
$$


for some $C_{1}>0$. Therefore, $u$ is a least energy sign-changing solution to problem (1.1).

Acknowledgments. The authors are grateful to the anonymous reviewers for their careful reading the manuscript and giving valuable comments and suggestions. This work is supported by National Science Foundation of China (No. 11771468 and No. 11971027).

Conflict of interest: Authors state no conflict of interest.

\section{References}

[1] C.O. Alves, M.A.S. Souto, Existence of solutions for a class of nonlinear Schrödinger equations with potential vanishing at infinity, J. Differential Equations 254(2013), 1977-1991.

[2] A. Ambrosetti, On Schrödinger-Poisson systems, Milan J. Math. 76 (2008), 257-274.

[3] A. Ambrosetti, V. Felli, A. Malchiodi, Ground states of nonlinear Schrödinger equations with potentials vanishing at infinity, J. Eur. Math. Soc. 7 (2005), 117-144.

[4] A. Bahrouni, V.D. Radulescu, P. Winkert, A critical point theorem for perturbed functionals and low perturbations of differential and nonlocal systems, Adv. Nonlinear Stud. 20 (2020), 663-674.

[5] R. Benguria, H. Brezis, E. Lieb, The Thomas-Fermi-von Weizäscker theory of atoms and molecules, Comm. Math. Phys. 79 (1981), 167-180.

[6] O. Bokanowski, J.L. López, J. Soler, On an exchange interaction model for quantum transport: The Schrodinger-PoissonSlater sytem, Math. Models Methods Appl. Sci. 13 (2003), 1397-1412.

[7] H. Brézis, E. Lieb, A relation between pointwise convergence of functions and convergence of functionals, Proc Amer Math Soc. 88 (1983), 486-490.

[8] G. Cerami, G. Vaira, Positive solutions for some non-autonomous Schrödinger-Poisson systems, J. Differential Equations 248(2010), 521-543.

[9] J.M. do Ó, O.H. Miyagaki, M. Squassina, Critical and subcritical fractional problems with vanishing potentials, Commun. Contemp. Math. 18(2016), 1550063, 20.

[10] M. Ghergu, G. Singh, On a class of mixed Choquard-Schrödinger-Poisson system, Discrete Contin. Dyn. Syst. S 12 (2019), 297-309.

[11] N. Ghoussoub, C. Yuan, Multiple solutions for quasi-linear PDEs involving the critical Sobolev and Hardy exponents, Trans. Amer. Math. Soc. 352 (2000), 5703-5744.

[12] X. He, W. Zou, Existence and concentration of ground states for Schrödinger-Poisson equation with critical growth, J. Math. Phys. 53 (2012), No. 023702.

[13] H. Hofer, Variational and topological methods in partially ordered Hillbert spaces, Math. Ann. 261 (1982), 493-514.

[14] W. Huang, X. Tang, Semiclassical solutions for the nonlinear Schrödinger-Maxwell equations, J. Math. Anal. Appl. 415 (2014), 791-802.

[15] G. Li, S. Peng, S. Yan, Infinitely many positive solutions for the nonlinear Schrödinger-Poisson systems, Commun. Contemp. Math. 12 (2010), 1069-1092.

[16] E. Lieb, M. Loss, Analysis, American Mathematical Society; 2001.

[17] W. Liu, Existence of multi-bump solutions for the fractional Schrödinger-Poisson system, J. Math. Phys. 57 (2016), (9):091502.17.

[18] Z. Liu, J. Zhang, Multiplicity and concentration of positive solutions for the fractional Schrödinger-Poisson systems with critical growth, ESAIM Control Optim. Calc. Var. 23 (2017), 1515-1542.

[19] N.J. Mauser, The Schrodinger-Poisson- $X_{\alpha}$ equations, Appl. Math. Lett. 14 (2001), 759-763.

[20] C. Miranda, Un'osservazione su un teorema di Brouwer, Boll. Unione Mat. Ital. 3 (1940), 5-7.

[21] E.G. Murcia, G. Siciliano, Positive semiclassical states for a fractional Schrödinger-Poisson system, Diff. Integral Equations 30 (2017), 231-258.

[22] E.Di Nezza, G. Palatucci, E. Valdinoci, Hitchhiker’s guide to the fractional Sobolev spaces, Bull. Sci. Math. 136 (2012), 521 573.

[23] N.S. Papageorgiou, V.D. Radulescu, D.D. Repovs, Nonlinear analysis-theory and methods. Springer Monographs in Mathematics. Springer, Cham, 2019. $x i+577$.

[24] P.H. Rabinowitz, Minimax methods in critical point theory with applications to differential equations. Pub- lished for the Conference Board of the Mathematical Sciences, Washington (DC); Providence (RI): American Mathematical Society, 1986. (CBMS regional conference series in mathematics; 65).

[25] D. Ruiz, Semiclassical states for coupled Schrödinger-Maxwell equations concentration around a sphere, Mathematical Models and Methods in Applied Sciences 15(2005), 141-164. 
[26] R. Servadei, E. Valdinoci, The Brezis-Nirenberg result for the fractional Laplacian, Trans. Amer. Math. Soc. 367 (2015), 67 102.

[27] L. Silvestre, Regularity of the obstacle problem for a fractional power of the Laplace operator, Comm. Pure Appl. Math. 60 (2007), 67-112.

[28] J. Slater, A simplication of the Haree-Fock method, Phys. Rev. 81 (1951), 385-390.

[29] J. Sun, H. Chen, L. Yang, Positive solutions of asymptotically linear Schrödinger-Poisson systems with a radial potential vanishing at infinity, Nonlinear Analysis TMA 74 (2011), 413-423.

[30] J. Sun, T.-F. Wu and Z. Feng, Multiplicity of positive solutions for a nonlinear Schrödinger-Poisson system, J. Differential Equations 260 (2016), 586-627.

[31] J. Sun, H. Chen, J.J. Nieto, On ground state solutions for some non-autonomous Schrödinger-Poisson systems, J. Differential Equations 252 (2012), 3365-3380.

[32] K. Teng, R. P. Agarwal, Existence and concentration of positive ground state solutions for nonlinear fractional Schrödinger-Poisson system with critical growth, Math. Meth. Appl. Sci. 41 (2018), 8258-8293.

[33] K. Teng, Ground state solutions for the non-linear fractional Schrödinger-Poisson system, Appl. Anal. 66 (2018), 1-38.

[34] K. Teng, Existence of ground state solutions for the nonlinear fractional Schrödinger-Poisson system with critical Sobolev exponent, J. Differential Equations 261(2016), 3061-3106.

[35] K. Teng, Y. Cheng, Multiplicity and concentration of nontrivial solutions for fractional Schrödinger-Poisson system involving critical growth, Nonlinear Analysis TMA 202 (2021), 112144.

[36] K. Teng, Ravi P. Agarwal, Ground state and bounded state solution for the nonlinear fractional Choquard-SchrödingerPoisson system, J. Math. Phys. 60 (2019), 103507; doi: 10.1063/1.5052473.

[37] D. Wang, Y. Ma, W. Guan, Least energy sign-changing solutions for the frational Schrödinger-Poisson system in $\mathbb{R}^{3}$, Bound. Value Prob. 2019 (2019), No. 25.

[38] Z. Wang, H. Zhou, Sign-changing solutions for the nonlinear Schrödinger-Poisson system in $\mathbb{R}^{3}$, Calc. Var. Partical Differential Equations 52(2015), 927-943.

[39] L. Wen, S. Chen, V.D. Radulescu, Axially symmetric solutions of the Schrödinger-Poisson system with zero mass potential in $\mathbb{R}^{2}$, Appl. Math. Lett. 104(2020), 106244.

[40] M. Willem, Minimax Theorems, Birkhäuser, 1996.

[41] L. Xu, H. Chen, Multiplicity of small negative-energy solutions for a class of nonlinear Schrödinger-Poisson systems, Appl. Math. Comput. 243 (2014), 817-824.

[42] Z. Yang, Y. Yu, F. Zhao, Concentration behavior of ground state solutions for a fractional Schrödinger-Poisson system involving critical exponent, Commun. Contemp. Math. 2018;1-46. DOI:10.1142/S021919971850027X.

[43] Y. Yu, F. Zhao, L. Zhao, The existence and multiplicity of solutions of a fractional Schrödinger-Poisson system with critical growth, Sci China Math. 61 (2018), 1039-1062.

[44] Y. Yu, F. Zhao, L. Zhao, The concentration behavior of ground state solutions for a fractional Schrödinger-Poisson system, Calc. Var. Partical Differential Equations 56 (2017); 56(4). Art. 116,25.

[45] Y. Yu, F. Zhao, L. Zhao, Positive and sign-changing least energy solutions for a fractional Schrödinger-Poisson system with critical exponent, Appl. Anal. 99(2020), 2229-2257.

[46] W. Zou, Sign-Changing Critical Point Theory, Springer, New York, 2008.

[47] L. Zhao, F. Zhao, Positive solutions for Schrödinger-Poisson equations with a critical exponent, Nonlinear Analysis TMA 70(2009), 2150-2164. 